\title{
Electric Properties of Carboxymethyl Cellulose
}

\author{
Alexandar Metodiev Zhivkov \\ Additional information is available at the end of the chapter
}

http://dx.doi.org/10.5772/56935

\section{Introduction}

The linear polymer carboxymethyl cellulose (CMC) as a polyelectrolyte is an object of consideration in this review. The emphasis is on the electric properties of CMC both as a free chain in solution and adsorbed on the solid surface. A special attention is paid to the mobility of counterions, electrostatically associated with the CMC polyelectrolyte chain.

\section{Chemical structure}

\subsection{Molecular mass}

Carboxymethyl cellulose $\left[\mathrm{C}_{6} \mathrm{H}_{7} \mathrm{O}(\mathrm{OH})_{3}-x\left(\mathrm{OCH}_{2} \mathrm{COOH}\right)_{x}\right]_{n}$ is a derivative of the regenerated cellulose $\left[\mathrm{C}_{6} \mathrm{H}_{10} \mathrm{O}_{5}\right]_{\mathrm{n}}$ with hydroxy-acetic acid (hydroxy ethanoic acid) $\mathrm{CH}_{2}(\mathrm{OH}) \mathrm{COOH}$ or sodium monochloroacetate $\mathrm{ClCH}_{2} \mathrm{COONa}$. The $\mathrm{CMC}$ backbone consist of D-glucose residues linked by $\beta-1,4$-linkage. The molecular mass of one glucose unit in CMC chain is $m_{\mathrm{CMC}}=146.14+75.04 \cdot x$, where $x \leq 3$ is the degree of substitution (DS). As a rule CMC is produced as sodium salt $\left[\mathrm{C}_{6} \mathrm{H}_{7} \mathrm{O}(\mathrm{OH})_{3} x\left(\mathrm{OCH}_{2} \mathrm{COONa}\right)_{x}\right]_{\mathrm{n}}$ with $\mathrm{DS}=0.4-1.2$, then the molecular mass per unit is $m \mathrm{NaCMC}=146.14+97.03 \cdot x \approx 185-263 \mathrm{~g} / \mathrm{mol}$.

\subsection{Structure of the chain backbone}

As derivative of the cellulose (poly- $\beta$-D-glucose) $[1,2,3] \mathrm{CMC}$ has inherited its main structural peculiarities: (a) rigidity of the glucose units (6-atoms rings in "armchair" conformation); (b) an almost fully extended conformation owing to impossibility for rotation round the $\mathrm{C}-\mathrm{O}-\mathrm{C}$ bonds between the glucose residues because of strong steric limitations (a consequence of the $\beta$-configuration at C-1 atom in 1,4-linkage); and (c) orientation of the bigger constituent groups $\left(-\mathrm{OH},-\mathrm{CH}_{2} \mathrm{OH}\right.$ and $\left.-\mathrm{OCH}_{2} \mathrm{COOH}\right)$ in the equatorial plane out of the saccharic ring. The crosssectional dimension of the CMC chain is a sum of the diameter of the glucose ring $(0.5 \mathrm{~nm})$ and the size of the carboxymethyl group $(0.4 \mathrm{~nm}$, situated on the both side of the chain); the 
effective diameter of the chain is rather higher because the glucose residues are oriented not line in line but somewhat tilted along the backbone line [4]. The replacement of the H-atom of -OH-group with $-\mathrm{CH}_{2} \mathrm{COO}^{-}$causes a loss of the possibility of parallel association of $\mathrm{CMC}$ chains in sheet-like structures (a characteristic of the cellulose structure leading to supramolecular organization in microfibrils [5]) because of both steric hindrance and electrostatic repulsion but that does not exclude the possibility of intra- and intermolecular interaction by hydrogen bonds between the unsubstituted $-\mathrm{OH}$ groups.

In the literature the polymer unit of $\mathrm{CMC}$ chain is denoted as consisting of one or two glucose rings following the manner applying to the cellulose. The one unit way is chemically wellfounded according the cellulose contents because all the glucose units are chemically identical (except those on the two ends of the chain). According to the conformation of the cellulose chain a more thought-out manner is the two glucose residues unit because this way allows distinguishing the cellulose (poly- $\beta$-D-glucose) and the amylose (poly- $\alpha$-D-glucose), both linear chains of chemically identical glucose residues. The conformation of the polymer chains of these two polysaccharides differs drastically owing to the configuration at the anomeric C-1 atom: the $\beta$-1,4-linkage in the cellulose leads to inversed orientation of every second glucose ring according the previous one and to sterically conditioned full extended conformation because of impossible rotation round the $\mathrm{C}-\mathrm{O}-\mathrm{C}$ bonds between $\mathrm{C}-1$ and $\mathrm{C}-4$ atoms of the neighbour rings. In the case of the amylose the $\alpha$-configuration at $\mathrm{C}-1$ atom leads to uniform orientation of the glucose rings and to impossibility of extended conformation; due to the $\alpha-$ 1,4-linkage amylose chain has a tendency to spiral conformation [5]. Since the linear charge density of the $\mathrm{CMC}$ chain is determined by the number of $-\mathrm{COO}^{-}$groups per glucose unit independently of its orientation, in this chapter the one glucose residue is accepted to denote the monomeric unit.

\subsection{Distribution the carboxylic groups along the chain}

The $-\mathrm{CH}_{2} \mathrm{COOH}$ group can be attached to every of the three hydroxyl groups of the cellulose monomer unit, so theoretically the degree of substitution (DS) can reach 3, but usually DS does not exceed 2. The distribution of $-\mathrm{CH}_{2} \mathrm{COOH}$ groups in the glucose unit and along the CMC chain is accepted to be random in the model [4] proposed to give the monomer composition of a sample with known DS. The model is based on the supposition that the substitute reaction occurs at random, i.e. each of the three $-\mathrm{OH}$ groups (at $\mathrm{C}-2, \mathrm{C}-3$ and C-6 position) of every monomer unit can be substituted in equal probability independently on the presence of another constituent in the same glucose unit. The experimental investigations $[6,7,8]$ of the mole fraction of substituted monomers of CMC confirm the hypothesis for random distribution of the constituents. For example, at DS $=1.3$ the mole fractions are about 0.2, 0.4, 0.3 and 0.1 for unsubstituted, mono-, di- and trisubstituted, respectively. On the base of these results it can be concluded that the $-\mathrm{CH}_{2} \mathrm{COO}^{-}$groups are also randomly distributed along the $\mathrm{CMC}$ chain and practically there are no regions containing more than one or two uncharged monomer units when DS is higher than 1-2. The absence of long uncharged segments allows accepting that the negative charges are almost evenly distributed along the CMC chain at high linear charge density 
(high DS and high degree of dissociation); that is important condition for applying the model of uniformly charged cylinder to CMC chain.

\subsection{Contour length}

The distance between two glucose units in CMC chain is $0.515 \mathrm{~nm}$ [4]. The contour length $L_{c}$ (the length of the chain backbone if it is fully stretched but without deformation of the valence angles and bonds) is determined as a product of the number of monomers $n$ and the length of one unit: $L_{\mathrm{c}}=0.515 \cdot n(\mathrm{~nm})$.

For example, at DS $=1.20 \mathrm{CMC}$ chain with $M_{\mathrm{NaCMC}}=250 \mathrm{kDa}$ (sodium salt) has 952 glucose residues and counter length $L_{c}=490 \mathrm{~nm}$.

\section{Electric properties of CMC in aqueous medium}

In this section the characteristics of CMC as a free polyelectrolyte chain are overlooked, i.e. the chain has conformational freedom that does not or depends on the presence of other macromolecules, respectively in diluted solution or semi-concentrated ones. The chain is immersed in salt-free medium or in electrolyte with valency $z$. In the first case the counterions originate from the ionizable group of the chain: $\mathrm{H}^{+}$or $\mathrm{Na}^{+}$(in the case of $\mathrm{CMC}$ or its sodium salt $\mathrm{NaCMC}$ ). A part of counterions can be electrostatically adsorbed losing their freedom; the rest are scattered by the thermal energy $k T$ and are randomly distributed around the chain forming an ions cloud.

\subsection{Dissociation of the carboxylic groups in CMC chain}

\subsubsection{Apparent dissociation constant}

The effective dissociation constant $\mathrm{K}_{a}$ (respectively $\mathrm{pK}_{a}=-\log \mathrm{K}_{a}$ ) of a weak polyelectrolyte depends on the neighbour electric charges in the chain and in the medium. The presence of other negative charges in the vicinity of a given $\mathrm{COO}^{-}$group leads to increasing of the electric field intensity and the local concentration of $\mathrm{H}_{3} \mathrm{O}^{+}$ions. As a result the probability of protonation of the $\mathrm{COO}^{-}$group increases; respectively the equilibrium of the reaction $\mathrm{COOH} \leftrightarrow \mathrm{COO}^{-}+\mathrm{H}^{+}$is shifted to the non-dissociated form. That is why the dissociation capability of the ionizable groups in the polyelectrolyte chain can not be characterized by a constant like in the case of a simple (monomeric) acid ( $\mathrm{pK}_{a}=\mathrm{pH}$ at $\left.\alpha=1 / 2\right)$. The apparent (effective) dissociation "constant" $\mathrm{pK}_{a}=f(x, \alpha, \mu)$ of $\mathrm{COOH}$ groups in CMC chain is a function of the degree of substitution $x$, the degree of dissociation $\alpha$ (determined by $\mathrm{pH}$ of the medium), and the ionic strength $\mu$; the first two factors determine the linear charge density of the chain.

\subsubsection{Degree of dissociation}

The degree of ionization (the fraction of the dissociated acid groups) $\alpha$ of a weak polyelectrolyte $\mathrm{pK} a$ depends on the apparent dissociation constant $\mathrm{K}_{a}(\alpha)$ at given $\alpha$ and the $\mathrm{H}^{+}$concentration: 


$$
\log [\alpha /(1-\alpha)]=\mathrm{pH}-\mathrm{pK}_{a} .
$$

$\mathrm{K}_{a}$ can be represented as a sum of the intrinsic dissociation $\mathrm{K}_{a}$ and the electrostatic term $\Delta \mathrm{K}_{a}$ (owing to the influence of the neighbour electric charges):

$$
\mathrm{pK}_{a}=\mathrm{pK}_{a 0}+\Delta \mathrm{pK}_{a}
$$

At equilibrium the shift $\Delta \mathrm{pK}_{a}$ is due to an alteration of the electrostatic free energy $G_{n}$ of the polyacid at variation of $n$ negatively charged groups [9]:

$$
\Delta \mathrm{pK}_{a}=(0.434 / k T)\left(d G_{\mathrm{n}} / d n\right) .
$$

\subsubsection{Experimental data}

Potentiometric titration of CMC aqueous solutions shows that the degree of acid dissociation (ionization) $\alpha$ increases in the range of $\mathrm{pH} 2-6$. The fact is determined by the moderate value of the dissociation constant $\mathrm{K}_{a}$ of the $-\mathrm{COOH}$ groups and allows defining $\mathrm{CMC}$ as a weak polyelectrolyte.

An addition of a low molecular salt to the CMC solution leads to increasing of the degree of dissociation $\alpha$ because of the screening of the electric field around the polyelectrolyte chain; the influence of the salt increases with $\alpha$ and the degree of substitution $x$, i.e. with the linear charge density. For CMC with DS $x=0.75$ the degree of dissociation increases from $\alpha=0.23$ to 0.85 in the range $\mathrm{pH} 3.0-5.0$ in $0.01 \mathrm{~mol} / \mathrm{L} \mathrm{NaCl}$; at increasing of $\mathrm{NaCl}$ concentration up to $1 \mathrm{~mol} / \mathrm{L}$ the dissociation increases from $\alpha=0.43$ to 0.98 in the same $\mathrm{pH}$ diapason [8]. The increasing of the ionic strength from 0.01 to $1 \mathrm{~mol} / \mathrm{L}$ shifts $\mathrm{pK}_{a}$ form 3.8 to 3.2 ; i.e. the dissociation at $\alpha=0.5$ is quadruplicately increased due to the electrostatic screening by $\mathrm{Na}^{+}$ ions.

\subsubsection{Intrinsic dissociation constant}

For aqueous solutions of CMC the experimental dependence of $\mathrm{pK}_{a}$ on the degree of dissociation $\alpha$ is linear with a slope $\Delta \mathrm{pK}_{a} / \Delta \alpha$ :

$$
\mathrm{pK}_{a}=\mathrm{pK}_{a 0}+\left(\Delta \mathrm{pK}_{a} / \Delta \alpha\right) \alpha .
$$

That allows obtaining the intrinsic dissociation constant $\mathrm{pK} a 0$ by extrapolation to $\alpha=0$ on the plot $\mathrm{pK}_{a}=f(\alpha)$. Thus the extrapolated values are: $\mathrm{pK}_{a 0} \approx 3.3$ in salt-free solution of CMC [10]; $\mathrm{K}_{a 0} \approx 3.2$ in $\mathrm{NaCl}$ solution with concentration $0.01,0.05$ and $0.1 \mathrm{~mol} / \mathrm{L} ; \mathrm{K}_{a 0} \approx 3.0$ in $0.5 \mathrm{~mol} / \mathrm{L}$ and $\mathrm{K}_{a 0} \approx 2.95$ in $1 \mathrm{~mol} / \mathrm{L} \mathrm{NaCl}[8]$.

It is interesting to compare the $\mathrm{K}_{a 0}$ of $\mathrm{CMC}$ with $\mathrm{K}_{a}$ of the acetic acid in diluted aqueous solution (when the electrostatic interactions between the next $-\mathrm{COO}^{-}$groups are negligible because of the big distance between acid molecules just in the opposite to the case of the polyelectrolyte chain). The thermodynamic acidity constant $\mathrm{K}_{a}$ of $\mathrm{CH}_{3} \mathrm{COOH}$ in aqueous medium is equal to $1.754 \cdot 10^{-5}$ (calculated accounting the activity instead the concentration), 
thus $\mathrm{pK}_{a}=4.756$ (the negative logarithm of $\mathrm{K}_{a}$ ). So, the intrinsic dissociation constant $\mathrm{K}_{a 0}$ of the carboxylic groups of $\mathrm{CMC}$ (at $\mathrm{pK} a 0 \approx 3.0$ in $0.5 \mathrm{~mol} / \mathrm{L} \mathrm{NaCl}$ ) is about 60 times higher than $\mathrm{K}_{a}$ of the acetic acid $-\mathrm{COOH}$ group. This observation is opposite to the expectation $\left(\mathrm{K}_{a 0}=\mathrm{K}_{a}\right.$ at $\alpha \rightarrow 0$ and $\mathrm{K}_{a 0}<\mathrm{K}_{a}$ when $\alpha>0$ ) if taking into account only the electrostatic interactions between the neighbour $-\mathrm{COO}^{-}$groups in the $\mathrm{CMC}$ chain. The difference in $\mathrm{K}_{a}$ and $\mathrm{K}_{a}$ can be explained with the different electronic structure of the two chemical substances. That suggest that the intrinsic dissociation constant of the three $-\mathrm{COOH}$ groups are different, especially of that directly attached to the glucose rings at C-2 and C-3 atoms and that at C-6 position.

\subsubsection{Theoretical approach}

The $\mathrm{pH}$-induced shift of the apparent dissociation constant $\Delta \mathrm{K}_{a}$ determined by the change of the electrostatic free energy $G_{n}$ of the polyion can be calculated by the work needed to display the elementary positive charge (proton) $q_{\text {e }}$ from the polyacid surface (where the electric potential is $\psi_{0}$ ) to a infinite distance in the bulk (where $\psi_{\infty}=0$ by definition). Then $(d G n / d n)$ in Eq. (3) effectively equals to $q_{\mathrm{e}} \psi 0$.

One of the models used for this calculations is the that of uniformly charged cylinder with radii $a<b$ ( $2 a$ is the mean diameter of the polyelectrolyte chain and $2(b-a)$ is the size of the adsorbed counterions). The $\Delta \mathrm{K}_{a}$ is proportional to the alteration of the electric potential owing to both the adsorbed (condensed) and diffuse counterions of all kinds $\left(\mathrm{Na}^{+}\right.$in the case of $\mathrm{NaCMC}$ ), represented by the potentials $\psi_{0}$ of the inner and $\psi_{1}$ of the external cylinder. The potential $\psi_{0}$ of the inner cylinder is determined by the degree of substitution $x$ and the degree of dissociation $\alpha$; the $\psi_{1}$ potential can be calculated from the surface charge density of the inner cylinder and the capacity of a cylinder capacitor with radii $b>a$ and interelectrode distance $b-a$. Then the shift $\Delta \mathrm{pK}$ a can be represented as a sum of two terms determined by the charge density of the inner cylinder and the potential on the external one [11]:

$$
\Delta \mathrm{pK}_{\mathrm{a}}=\left(0.217 e^{2} / \pi \varepsilon_{0} \varepsilon_{\mathrm{r}} k T\right) \ln (b / a) x \alpha+(0.434 e / k T) \psi_{1},
$$

where $e$ is the elementary charge (proton); $\varepsilon_{0} \varepsilon_{\mathrm{r}}$ is the absolute dielectric permittivity (electric constant $\varepsilon_{0}$ and the relative dielectric permittivity $\varepsilon_{\mathrm{r}}$ ); $k T$ is the thermal energy at absolute temperature $T$ ( $k$ is the Boltzmann constant).

In the case of $\mathrm{NaCMC}$ the Eq. (5) takes into account the two kinds of counterions: $\mathrm{H}^{+}$ions determining the potential $\psi_{0}$ of the inner cylinder and adsorbed $\mathrm{Na}^{+}$cations determining $\psi_{1}$ of the external one. By fitting the experimental data for the degree of dissociation $\alpha=f(\mathrm{pH})$ (obtained by titration of $\mathrm{CMC}$ solution with $\mathrm{NaOH}$ ) with the parameters of the model of uniformly charged cylinder in Ref. [8] are found the intrinsic dissociation constant $\mathrm{K}_{a 0}=3.2$ (at $0.01-0.1 \mathrm{~mol} / \mathrm{L} \mathrm{NaCl}$ ) and radius of the inner cylinder $a \approx 1 \mathrm{~nm}$; the last quantity increases from $a=0.95$ to $1.15 \mathrm{~nm}$ when the degree of substitution grows from $x=0.75$ to 1.25 . So, the calculated diameter $2 a \approx 2 \mathrm{~nm}$ is two times higher than that of the molecular cross-section of $\mathrm{CMC}$ chain if taken as a sum of the size the glucose ring $(0.5 \mathrm{~nm})$ and one attached $\mathrm{COOH}$ group $(0.4 \mathrm{~nm})$. 


\subsection{Linear charge density of CMC chain}

The dissociation of carboxylic groups in aqueous medium determines the electric properties of $\mathrm{CMC}$ as polyelectrolyte. The number of $\mathrm{COO}^{-}$groups is determined by the degree of substitution (DS) and the degree of dissociation $\alpha$. The linear charge density $b^{-1}$ is equal to the ratio of ionized groups' number to the contour length $L_{c}$. For example, CMC chain with DS $=1.20$ and $M=250 \mathrm{~kg} / \mathrm{mol}$ (sodium salt) has 952 residues, $L_{c}=490 \mathrm{~nm}$ and mean distance between two neighbour $\mathrm{COO}^{-}$groups $b=0.429 \mathrm{~nm}\left(b^{-1}=2.33\right.$ charges $\left./ \mathrm{nm}\right)$ at $\alpha=1$. For CMC with DS $=1.00(b=0.515 \mathrm{~nm})$ the charge density is $b^{-1}=1.94 \mathrm{~nm}^{-1}$ at full dissociation or nearly one charge per nanometer at $\alpha=1 / 2$.

So defined charge density is determined by the chemical structure of CMC chain and could be designate as intrinsic linear charge density; it takes into account only chain's own charges $\left(\mathrm{COO}^{-}\right.$groups). The presence of adsorbed (condensed) counterions $\left(\mathrm{Na}^{+}\right.$in the case of sodium salt of $\mathrm{CMC}$ ) decreases the effective charge density. The value of $\alpha$ obtained by potentiometric titration reflects all kinds of electrostatic interactions influencing on $\Delta \mathrm{K}_{a}$ including that of the condensed $\mathrm{Na}^{+}$counterions and the alteration of $\mathrm{H}^{+}$concentration in the vicinity of the polyelectrolyte chain. So, the using of experimental values of $\alpha$ gives the intrinsic linear charge density.

\subsection{Counterions condensation on CMC chain}

\subsubsection{Condensation phenomenon and theoretical approaches}

When a polyelectrolyte chain is immersed in aqueous solution of electrolyte the electrostatic field around the chain increases the local concentration of the diffuse counterions and decreases the one of the co-ions; the difference between the two concentrations is maximal at the chain surface and diminishes quasi-exponentially down to zero in the bulk. The distribution of the local concentration of the free ions is determined by the ratio between the electrostatic and the thermal energies. When this ratio increases above a critical value then part of counterions are electrostatically adsorbed on the chain forming temporary ion pairs with the electric charges of the polymer; the phenomenon is known as counterion condensation.

The distribution of the diffuse and condensed counterion considered as loosely and strongly associated with the polyion is a subject of several polyelectrolyte theories developed on the base of a few different models [12]; the most popular are: Katchalsky's cylindrical cell model [13] and the Oosawa-Manning two state model [14]. In the both models the polyelectrolyte chain is considered as an infinite impenetrable cylinder on which surface the charges are smeared as a non-discrete quantity and uniformly distributed along the cylinder axis.

The main imperfections of the cylindrical models are: (1) the real polyelectrolyte chain can be assumed as strait only for a rigid fragment shorter than the persistent length $l_{\mathrm{p}}$ and when the $l_{\mathrm{p}}$ is much longer than the chain diameter (the semi-flexible chains like CMC have a form of curved rod when their contour length is commensurable with $l_{\mathrm{p}}$ but the longer chains takes conformation of random coil); (2) the end-effects are neglected; that assumption is 
defensible only for enough long chains having big number of charges; (3) the charged groups are non-uniformly distributed along the chain backbone at incomplete degree of dissociation $(\alpha<1)$ and/or at irregular distribution of the dissociable groups as in the case of CMC (see paragraph 2.3); and (4) the chain's charges can be considered as non-discrete quantity uniformly smeared on the cylinder only at a some distance from the surface (where the electric field is less inhomogeneous); at a shorter distance the charges should be considered as discrete and distributed according to the chain structure.

\subsubsection{Cylinder cell model}

In this model $[15,16,17]$ a polyelectrolyte cylinder with radius $a$ is localized in the center of an infinitely long cylinder cell with radius $R$; the distance $(R-a)$ is divided into two zones. In the inner zone the centers of the adsorbed (condensed) counterions are uniformly distributed on a coaxial cylinder with radius $b$; the difference in radii $(b-a)$ of the two cylinders is equal to the ion radius. The electric potential $\psi_{0}$ (on the inner cylinder surface) is determined by the electric charges of the polyelectrolyte chain themselves. The potential in the inner zone (at distance $b-a$ ) follows lineally as in a homogeneous solid state body; that allows to consider the polyelectrolyte chain as a coaxial cylindrical condenser. The presence of two kinds of counterions with different radius and valence can be considered by introducing an additional cylinder in the inner zone $[18,19]$. The potential $\psi_{1}$ of the cylinder with radius $b$ is determined by the total chain's charge including the adsorbed ions. In the external zone $(R-b)$ the diffuse counterions are uniformly scattered according the balance between the electrostatic $e \psi_{1}$ and thermal energy $k T$; their size is assumed to be negligibly small (point charges). The potential in the external zone follows quasi-exponentially (in the case of symmetrical electrolyte in the medium) according the non-liner Poisson-Boltzmann equation in its cylinder form $[20,21]$.

So, the cylinder cell model is a one-dimensional analog of the two-dimensional electric double layer (EDL) used in the colloid chemistry to describe the electric properties of a flat solid/liquid interface: the two inner cylinders are analogs of the solid surface and Stern layer (the EDL dense part). The difference is that at low potential ( $\psi_{1}<25 \mathrm{mV}$ in 1:1 electrolyte) $\psi(x)$ follows exponentially with the distance $x$ in the case on flat surface while in the case of cylinder the potential distribution $\psi(x)$ is quasi-exponential. The main advantage of the cylindrical cell model is that it gives the radial counterions distribution around the polyelectrolyte chain; that is especially important in salt solutions. But its application is difficult because in the most cases it requires numerical solution of the non-liner PoissonBoltzmann equation; the exact analytical solution is possible only for an infinite cylinder.

The application of the cylindrical cell model to NaCMC takes into account the two kinds of counterions $\left(\mathrm{H}^{+}\right.$and $\left.\mathrm{Na}^{+}\right)$in their two states (adsorbed and diffuse): $\mathrm{H}^{+}$ions determine the potentials of the inner $\left(\psi_{0}\right)$ cylinder and $\mathrm{Na}^{+}$cations on the external $\left(\psi_{1}\right)$ one; and the both cations are in competition in the diffuse cloud determining the potential $\psi(x)$ together with the $\mathrm{OH}^{-}$anions. The difference between the two counterions is principal at the surface: $\mathrm{H}^{+}$ are potential determining for $\mathrm{COO}^{-}$groups but $\mathrm{Na}^{+}$are adsorbed (condensed); in the diffuse 
cloud this difference disappear: $\mathrm{H}_{3} \mathrm{O}^{+}$and $\mathrm{Na}^{+}$are in competition determined by their concentration in the bulk.

\subsubsection{Bjerrum length}

The dependence of electrostatic energy on the distance between two ions (a negative and positive) leads to condition for equality of the electrostatic and thermal energies; the critical distance is called Bjerrum length:

$$
l_{\mathrm{B}}=e^{2} / 4 \pi \varepsilon_{0} \varepsilon k T
$$

where $e$ is the elementary charge (electron); $\varepsilon_{0} \varepsilon$ is dielectric permittivity of the medium (the relative permittivity $\varepsilon$ and electric constant $\varepsilon_{0}$ in SI); $k T$ is the thermal energy (the Boltzmann constant $k$ and the absolute temperature T). In aqueous medium: $l_{\mathrm{B}}=0.712 \mathrm{~nm}$ at $20^{\circ} \mathrm{C}(\varepsilon=$ $80.08)$ and $l_{\mathrm{B}}=0.700 \mathrm{~nm}$ at $25^{\circ} \mathrm{C}(\varepsilon=78.25)$. The Bjerrum length is about 3.8 times higher than the crystal radius of $\mathrm{Na}$ atom and commensurable with the diameter of hydrated $\mathrm{Na}^{+}$ion (the counterion in salt-free solution of $\mathrm{NaCMC}$ ).

Although Bjerrum had formulated the length $l_{\mathrm{B}}$ as a criterion for ion pair formation in a concentrated electrolyte solution, Eq. 6 is used in the Oosawa-Manning two-state polyelectrolyte theory for estimation of the electrostatic interactions between the polymer charges and small counterions.

\subsubsection{Two-state model}

In this model the counterions (electrostatically associated to the polyelectrolyte chain) are separated into two categories according their physical state: condensed (strongly bound and located inside a narrow potential valley along the polymer backbone) and free counterions (loosely bound and freely moving in the volume surrounding the chain). On the base of this model Manning has developed a simple theory for counterion condensation on isolated infinitively long cylinder immersed in a liquid medium with a vanishingly low ion concentration [22]. Such conditions appear in aqueous solutions of rigid or semi-flexible chains as DNA and CMC at low polymer concentration in salt free solution (counterions originate from the polymer sample: $\mathrm{Na}^{+}$in the case of $\mathrm{NaCMC}$ ).

The Manning's model consider the polyelectrolyte chain as a liner polyion with average distance $b_{0}$ between its charges surrounded by a cylinder with radius $a$. The main parameter is the dimensionless charge parameter $\xi$ determined by the linear charge density $b_{0}{ }^{-1}$ and the Bjerrum length $l_{\mathrm{B}} \approx 0.7 \mathrm{~nm}$ (at room temperature):

$$
\xi \equiv l_{\mathrm{B}} / b_{0}=e^{2} / 4 \pi \varepsilon_{0} \varepsilon k T b_{0} .
$$

A counterions condensation appears when $\xi>1$, i.e. when the mean distance between the two neighbour charges of the polyelectrolyte chain is smaller than $0.7 \mathrm{~nm}$. When the degree of dissociation $\alpha$ increases until $\xi<1$, the linear charge density $b^{-1}=N / L_{c}$ (determined by the 
total number $N$ of unit charges per unit contour length $L_{c}$ ) increases linearly up to a limit where $b_{0}=l_{\mathrm{B}}$; in this region $b=b_{0}$. At the point $\xi=1$, the liner dependence $b^{-1}=f(\alpha)$ is broken and goes as a plateau at $\xi>1$, then $N=$ const. I.e. at $\xi>1$ the effective charge density $b^{-1}$ has its maximal value for a given linear polyelectrolyte and does not increase more although the chain's own charges $b_{0}$ continue to increase with $\alpha$; this constancy of the total charge $N$ is due to counterion condensation [23].

The fraction $\varphi$ of the chain's charges neutralized by the condensed counterions with valency $z$ is equal to:

$$
\varphi=1-1 / z \xi=1-b_{0} / z l_{\mathrm{B}}
$$

This equation allows determining the quantity of the condensed counterions when the quantity of the chain's own charges (per unit contour length) is known. Like so the condensed ions fraction is determined by the distance between the ionizable groups along the chain and the degree of dissociation $\alpha$.

By that way the Manning's theory connect the counterions condensation with the chemical structure of the polyelectrolyte chain (the length of the polymer units and the nature of the ionizable groups) and the conditions of the medium ( $\mathrm{pH}$ and temperature) together determining the quantity of chain's own charges (equal to the total counterions quantity). The theory allows defining two fractions of counterions having different mobile capability: the condensed ions are immobile (during the existence of the Bjerrum pairs) while the 'free' ones are mobile undergoing impacts with the solvent molecules.

The Manning's theory does not give exact value of the condensed ions fraction even when its two limits of validity (an infinitely long cylinder and vanishingly small concentration of small ions) are satisfied because Eq.(8) is derived on the base of analytical solution of the cylindrical Poisson-Boltzmann equation in its linearized form (assuming $\sinh \psi \approx \psi$ ) [24]. The numerical calculation of the non-linear cylindrical Poisson-Boltzmann equation shows that at small $\xi$ (up to $\xi \approx 1 / 2)$ the liner dependence $b^{-1} \equiv N / L_{c}=f(\alpha)$ coincides with the Manning's line but it is not broken at $\xi=1$ and does not go on a plateau after this point. At $\xi>1$ the curve continue to increase although its slope decreases with $\xi$; this discrepancy increases with the ionic strength [8]. That means that the condensed ions fraction is smaller than the predicted by the Manning's theory. Nevertheless its experimental verification has shown an excellent agreement between the theoretical and experimental values in the case of double stranded DNA: at predicted $\varphi=0.76$ the obtained by NMR condensed counterions fraction is 0.75 [25].

\subsubsection{Application of Manning's theory to CMC}

The distance between two glucose units in CMC chain $(0.515 \mathrm{~nm})$ and the degree of dissociation $\alpha$ determine the liner charge density $b_{0}{ }^{-1}$. For $\mathrm{CMC}$ with degree of substitution DS $=1.00$ the limit $(\xi=1)$ for the ions condensation appears at $\alpha=0.72$ according to Manning's theory. At full dissociation $(\alpha=1)$ the charge density is $b_{0}{ }^{-1}=1.94 \mathrm{~nm}^{-1}$ and the dimensionless parameter is $\xi=1.38\left(\right.$ at $20^{\circ} \mathrm{C}$ ), so condensation of $\mathrm{Na}^{+}$counterions on $\mathrm{COO}^{-}$ 
groups appears even in salt-free solution of $\mathrm{NaCMC}$; the fraction of the condensed counterions is $\varphi=1-1 / \xi=0.28$.

For $\mathrm{CMC}$ with $\mathrm{DS}=1.20$ the mean distance between two $\mathrm{COOH}$ groups is equal to $0.43 \mathrm{~nm}$, so the charge density parameter is $\xi=1.66$ at $\alpha=1$. At $\mathrm{pH} 6.0$ the degree of dissociation is $\alpha$ $\approx 0.95[8], \xi=1.58$ and the condensed counterions fraction is equal to $\varphi=0.37$. So, the CMC chain remains negatively charged when it is immersed in salt-free aqueous medium at $\mathrm{pH}$ 6.0 ; about $63 \%$ of the dissociated groups are compensated by the diffuse counterions in the ionic atmosphere.

\subsubsection{Relation between condensation and dissociation}

The apparent dissociation constant $\mathrm{pK} a$ of the acid groups is shifted with $\Delta \mathrm{pK}_{a}$ relatively to its intrinsic value $\mathrm{pK}_{a 0}$ due to the increased local concentration of $\mathrm{H}_{3} \mathrm{O}^{+}$ions because of the strong electric field around the polyelectrolyte chain; the value of $\Delta \mathrm{pK} a$ is proportional to the charge density $b_{0}{ }^{-1}(\alpha)$ determined by the degree of dissociation $\alpha$ (Eq.3). It is reasonable to suppose that $\Delta \mathrm{pK}_{a}$ depends on counterions condensation both indirectly (by the effective value of $b^{-1}$ ) and directly (forming ions pair $\left[\mathrm{COO}^{-} \mathrm{Na}^{+}\right]$instead $\mathrm{COOH}$ and influencing on the polarization of $\mathrm{O}-\mathrm{H}$ electron-pair link in $\mathrm{COOH}$ groups). This means that the dependence of $\Delta \mathrm{pK}_{a}$ on $\alpha$ should have different course at $\xi<1$ and at $\xi>1$. This hypotheses can be experimentally tested by determining $\alpha=f(\mathrm{pH})$ and $\mathrm{pK}_{a}=f(\alpha)$ by potentiometric titration of aqueous solution of CMC.

For CMC with DS $=0.80$ and 0.90 the charge parameter $\xi$ is equal to 1 at $\alpha=0.90$ and 0.80 ; so, at these values of $\alpha$ the dependence $\mathrm{pK}_{a}(\alpha)$ should change its slope. This effect must be stronger at high ionic strength where the counterion condensation increases. Although in the last case the Manning's theory is inapplicable, the limiting value $\xi=1$ also can be used as the dependence $b^{-1}(\alpha)$ begins to deflect from line in the region of $\xi \approx 1$ as the numerical solution of the non-linear Poisson-Boltzmann equation shows [8].

The potentiometric titration $\alpha=f(\mathrm{pH})$ [8] shows that in salt-free solution the dependence $\mathrm{pK} a$ $=f(\alpha)$ is almost linear in the range $\alpha=0.1-0.9$ for CMC with DS $=0.81$. The addition of $\mathrm{NaCl}$ up to $1 \mathrm{~mol} / \mathrm{L}$ decreases the slope of the dependences $\mathrm{pK}_{a}(\alpha)$ but they are absolutely liner in the studded interval $\alpha=0.25-0.97$ for CMC with DS $=0.91$.

The absence of a curvature of the experimental dependence $\mathrm{pK}_{a}(\alpha)$ at $\xi \approx 1$ shows out that counterions condensation does not influence the degree of dissociation (in the frame of the experimental accuracy). This result can be explained with the different nature of $\mathrm{H}^{+}$and $\mathrm{Na}^{+}$ binding to $\mathrm{COO}^{-}$groups: the proton is involved in covalent linkage (coupled electron pair between $\mathrm{H}$ and $\mathrm{O}$ atoms) while the sodium ion is electrostatically bound. The different energy of these two kinds of banding leads to longer life of the $\mathrm{COO}-\mathrm{H}$ links in comparison to the $\mathrm{COO}^{-} \mathrm{Na}^{+}$bonds and respectively to much higher affinity of $\mathrm{COO}^{-}$groups to $\mathrm{H}^{+}$than to $\mathrm{Na}^{+}$ion.

Nevertheless, the presence of $\mathrm{Na}^{+}$ions in the medium influences the $\mathrm{COOH} \leftrightarrow \mathrm{COO}^{-}+\mathrm{H}^{+}$ equilibrium by the local concentration of $\mathrm{H}^{+}$in the diffuse cloud where $\mathrm{H}_{3} \mathrm{O}^{+}$and $\mathrm{Na}^{+}$ions are 
in competition being electrostatically equivalent; the evidence for that is the decreasing slope of the dependence $\mathrm{pK}_{l}(\alpha)$ at increasing concentration of $\mathrm{NaCl}$ [8]. This fact together with the absence of visible influence of $\mathrm{Na}^{+}$condensation on $\alpha$ shows out that the physical state of the counterions (immobile or free moving) is not as important as its concentration in the vicinity of the polyelectrolyte chain. This conclusion demonstrates the advantages of the cylindrical cell model (distinguishing the counterions by their distance from the surface and considering their local concentration) in comparison with the two-state model (separating the counterions into two categories according their motility and disregarding the 'free' counterions distribution).

\section{Conformation of free CMC chain in aqueous medium}

As a polyelectrolyte the conformation of CMC chain is determined by its chemical structure and electric properties determining the chain rigidity and linear charge density; the both quantities also depend on the medium parameters ( $\mathrm{pH}$, ionic strength and temperature). The electrostatic interactions could be neglected at low $\mathrm{pH}$ and/or high salt concentration; then the chain could be considered as a non-charged.

The main structural peculiarity of the cellulose and its derivatives are the rigidity of the glucose rings and the impossibility of rotation around the $\mathrm{C}-\mathrm{O}-\mathrm{C}$ bonds between the neighbour rings because of $\beta$-1,4-linkage; the both factors determine the rigidity of the chain backbone. Although very restricted oscillations round the $\mathrm{C}-\mathrm{O}-\mathrm{C}$ bonds occur and that decrease orientational correlation of the monomer units (regarding the line defined by the first init). That means that the orientation of every glucose unit strongly dependents on the orientation of significant number its neighbours but the orientational correlation decreases slowly along the chain. So, the CMC chain can be defined as a semi-flexible; that allows applying the model of worm-like chain.

\subsection{Theoretical definitions}

The conformation of an individual polymer chain is described by its end-to-end distance $h$ (the distance between the first and the last polymer units) or by radius of gyration $\mathrm{Rg}$ (averaged mean-square distance between the units and the center of mass); these quantities are averaged in time for one chain or for an ensemble of chains at aiven moment: $\langle h\rangle$ and $\left\langle R_{\mathrm{g}}\right\rangle$. The CMC chain with contour length $L_{\mathrm{c}}$ has $n=L_{\mathrm{c}} / l$ units with length $l=0.515 \mathrm{~nm}$. The value of $l$ is taken equal to the size of the glucose residue (instead the length of $C-C$ bound as in the case of flexible polymers) because of the rigidity of the glucose rings.

\subsubsection{Unperturbed random coil}

In the model of free-articulated chain (Kuhn's model) the real chain is replaced by a hypothetical chain with length $L_{\mathrm{c}}=A_{\mathrm{K}} N_{\mathrm{K}}$ consisting $N_{\mathrm{K}}>1$ segments with length $A_{\mathrm{K}}=s l$ (every segment has $s$ monomeric units with length $l$ ); the rotation between the neighbour segments is completely free. For an unperturbed random coil the Kuhn's theory gives for the mean-square end-to-end distance $\left\langle h^{2}\right\rangle$ : 


$$
\left\langle h^{2}\right\rangle_{0}=A_{\mathrm{K}} L_{\mathrm{c}}
$$

and for the statistical segment length:

$$
N_{\mathrm{K}}=L_{\mathrm{c}}^{2} /\left\langle h^{2}\right\rangle_{0}
$$

This equation allows determining $A_{\mathrm{K}}$ and $N_{\mathrm{K}}$ from the experimentally measured molecular mass $M \sim L_{c}$ and $\left\langle h^{2}\right\rangle_{0}$ or the mean-square radius of gyration $\left\langle R_{\mathrm{g}}{ }^{2}\right\rangle$. For an unperturbed random coil at $N_{K}>>1$ :

$$
\left\langle R_{\mathrm{g}}^{2}\right\rangle_{0}=\left\langle h^{2}\right\rangle_{0} / 6=\left(A_{\mathrm{K}} / 6\right) L_{\mathrm{c}} .
$$

The distribution of the segments of an unperturbed random coil is Gaussian at (a) $L_{\mathrm{d}} / A_{\mathrm{K}}>10$ and (b) when the polymer is immersed in "ideal solvent" $(\theta$-solution or $\theta$-temperature when the interactions polymer-polymer, polymer-solvent and solvent-solvent are compensated); for such coil $\left\langle h^{2}\right\rangle_{0}$ and $\left\langle R_{\mathrm{g}}{ }^{2}\right\rangle_{0}$ are proportional to the couture length $L_{c}$ according the Eqs. (9) and (11) [26].

\subsubsection{Expanded random coil}

Out of the $\theta$-conditions the proportionality between $\left\langle h^{2}\right\rangle,\left\langle R_{\mathrm{g}}{ }^{2}\right\rangle$ and $L_{c}$ is infringed but the chain can be return to conformation of unperturbed random coil changing the thermodynamic properties of the solvent by the temperature and addition of salts; an indication for $\theta$-point is an absence of dependence of $\left\langle h^{2}\right\rangle$ or $\left\langle R_{\mathrm{g}}{ }^{2}\right\rangle$ on the polymer concentration.

The linear expansion factor $\alpha_{e}$ is determined by the ratio between the random coil size (averaged end-to-end distance $\left\langle h^{2}\right\rangle^{1 / 2}$ or radius of gyration $\left\langle R_{\mathrm{g}}^{2}\right\rangle^{1 / 2}$ ) and its unperturbed value:

$$
\alpha_{\mathrm{e}}=\left\langle h^{2}\right\rangle^{1 / 2} /\left\langle h^{2}\right\rangle_{0}^{1 / 2}=\left\langle R_{\mathrm{g}}{ }^{2}\right\rangle^{1 / 2} /\left\langle R_{\mathrm{g}}{ }^{2}\right\rangle_{0}^{1 / 2} .
$$

In "good solvent" $\left(\alpha_{e}>1\right)$ or "bad solvent" $\left(\alpha_{e}<1\right)$ the chain has more expended or compact conformation, respectively. The dependence of $\alpha_{e}$ on the medium conditions is explained by the term "excluded volume" (equal to zero in "ideal solvent") which includes the physical volume of the chain and the difference of repulsion and attraction forces between polymer segments when distant chain units encounter owing their thermal motion; the probability of such collisions increases with the chain length and decreases with the rigidity of the chain: $\alpha_{\mathrm{e}} \sim L_{\mathrm{c}} / A_{\kappa}$. The excluded volume additionally increases in the case of polyelectrolytes (at low polymer concentration) because of the electrostatic repulsion between the distant intermolecular chain units.

\subsubsection{Persistent length}

At $L_{c} / A_{\kappa}<10$ the proportionality between $\left\langle h^{2}\right\rangle,\left\langle R_{\mathrm{g}}{ }^{2}\right\rangle$ and $L_{\mathrm{c}}$ is infringed (the segment distribution is non-Gaussian even in $\theta$-solvent), so the Eqs. 9-12 are inapplicable. To describe 
the conformation of such short and rigid chains the worm-like chain model is introduced; its peculiarity is the continuously changing chain curvature.

The averaged angle $\langle\varphi\rangle_{L}$ between the tangents to two points at distance $L_{1}$ along a worm-like chain is given by the expression:

$$
\cos \langle\varphi\rangle_{L}=\exp \left(-L_{1} / l_{p}\right)
$$

where the persistent length $l_{p}$ describes the $e$ fold decreasing of the orientational correlation. In such way $l_{p}$ is a measure for the rigidity (local stiffness) of the worm-like chain; at $L_{c} \leq l_{p}$ the chain can be described as a more or less curved rod. Comparison with the Kuhn model (at $\left.N_{K}>1\right)$ gives the ratio: $l_{p}=(1 / 2) A_{K}$.

The unperturbed radius of gyration $\left\langle R_{\mathrm{g}}{ }^{2}\right\rangle_{0}{ }^{1 / 2}$ can be expressed by the ratio between the persistent and contour lengths $y=l_{p} / L_{c}$ :

$$
\left\langle R_{\mathrm{g}}^{2}\right\rangle_{0}=L_{\mathrm{c}}^{2}\left\{(1 / 3) y-y^{2}+2 y^{3}-2 y^{4}[1-\exp (-1 / y)]\right\} \text {. }
$$

This equation allows describing the size of chins of all length and rigidity including the both limits: rigid rod (very shot and rigid chains, then $\left.\left\langle R_{\mathrm{g}}\right\rangle_{0}=(1 / 12) L c^{2}\right)$ and Gaussian coil (long and flexible chains at $L_{\mathrm{c}} / l_{p}>20$ in $\theta$-solvent).

The random coil expansion in good solvent leads to increase of the effective persistent length $l_{\mathrm{p}}=l_{\mathrm{p} 0}+l_{\mathrm{pe}}$, where the two terms represent the intrinsic persistent length and the electrostatic component, respectively. The electrostatic term decreases with the ionic strength of the medium; that manifest itself as decreasing of the random coil size $\left(\left\langle h^{2}\right\rangle^{1 / 2}\right.$ or $\left.\left\langle R_{\mathrm{g}}\right\rangle^{1 / 2}\right)$ when the salt concentration of the polymer solution increases.

\subsection{Experimental methods}

The classical techniques for experimental investigation of the polymer chain conformation in liquid medium are those of the viscometry and the static light scattering.

\subsubsection{Viscometry}

The viscometry is the technique most frequently employed in physical chemistry of polymer solutions. The method is based on the measure of the dynamic viscosity of the solvent $\eta_{0}$ and of polymer solution $\eta$ with concentration $c$ and extrapolation of the relative viscosity $\eta / \eta_{0}$ to $c=0$. The characteristic viscosity (the limiting viscosity number) defined as:

$$
[\eta]=\lim _{\mathrm{c} \rightarrow 0}\left[\left(\eta / \eta_{0}-1\right) / c\right]
$$

is connected with the molecular mass $M$ and the end-to-end size $\left\langle h^{2}\right\rangle_{0}^{1 / 2}$ of the macromolecules:

$$
[\eta]=K M^{a}, \text { and } \quad[\eta]=\Phi\left\langle h^{2}\right\rangle^{3 / 2} / M
$$


where $K$ and $a$ are constants specific for a given polymer/solvent solution; $\Phi \approx 2.1 \cdot 10^{23}$ (when the dimensions of $[\eta]$ and $\left\langle h^{2}\right\rangle_{0}^{1 / 2}$ are $\mathrm{cm}^{3} / \mathrm{g}$ and $\mathrm{cm}$, respectively) is a unspecific constant for linear chains with conformation of random coil in good solvent.

The constant $a$ gives information about the form of the macromolecules: $a=1.8$ for rigid thin rod with axes ratio $\geq 100$; for unperturbed random coil $a=0.5$ and increases up to 0.8 for an expanded coil. The method is widely used due to its experimental simplicity but it is not absolute requiring calibration with monodisperse fractions with independently determined molecular mass $M$. Once the constant $K$ and $a$ are known the equations (16) allows determining the mass and size of the random coil by measuring [ $\eta]$.

\subsubsection{Small angle static light scattering}

The second widely used method for determination of the polymer chain mass and conformation is the static light scattering [27]. Its main advantage is that it is absolute: for determination of the squared radius of gyration $\left\langle\mathrm{Rg}^{2}\right\rangle$ and molecular mass $M$ a calibration with polymer fractions is not required. Because the relative size $\left(\left\langle\mathrm{Rg}^{2}\right\rangle^{1 / 2} / \lambda\right.$ is small relatively to the wavelength $\lambda$ (in the medium), the small angle approximation (equation of Guinier) is applicable for the light scattering of polymer solutions with weight concentration $c$ :

$$
c H / R_{\theta}=1 / M+\left(16 \pi^{2} / 3\right)\left(n_{0} / \lambda_{0}\right)^{2}\left\langle R_{\mathrm{g}}{ }^{2}\right\rangle \tilde{n} \sin ^{2}(\theta / 2),
$$

where $H \sim\left[\left(n 0^{2} / \lambda_{0}{ }^{4}\right)(d n / d c)^{2}\right]$ is an optical constant (specific for a given pair polymer/solvent but not depending on $c$ in diluted solution) determined by the refractive indexes of the polymer solution $n$ and the medium $n_{0}$ at wavelength $\lambda_{0}$ (in vacuum); the increment of the refractive index $(d n / d c)=\left(n-n_{0}\right) / c($ at $c \rightarrow 0)$ is measured by refractometry. The scattering coefficient (Rayleigh ratio) $R_{\theta}=I / I_{0}$ is determined by the quantities measured in the experiment: intensities of the incident light $I_{0}$ and the scattered $I$ at angle $\theta$. For determination of $\left\langle R_{\mathrm{g}}{ }^{2}\right\rangle$ it is not required to know the absolute values of $c$ and $H$ : it is enough to measure the dependence $1 / I_{\theta} \sim \sin ^{2}(\theta / 2)$ at a few $c$ and extrapolate $1 / I_{\theta}$ to $c=0$. The extrapolated to $\theta=0$ concentration dependence $c / R_{0} \circ \sim A_{2} c$ (not considered in Eq. 17) gives the second virial coefficient $A_{2}$ which allows determination of the random coil expansion factor $\alpha_{e}[28]$.

\subsection{Factors determining CMC chain conformation}

\subsubsection{Rigidity and molecular mass}

The impossibility of rotation between the neighbour glucose units reflects in high persistent length $l_{\mathrm{p}}=5-20 \mathrm{~nm}$ of the cellulose and its derivatives [29]; this value is one-two orders higher than for polymers with flexible chains ( $l_{\mathrm{p}}=0.2 \mathrm{~nm}$ for poly(ethylene glycol) [26]). The relatively high value of the $l_{\mathrm{p}}$ allows to define $\mathrm{CMC}$ chain as worm-like and suggest that (depending on the contour length $L_{c}$ ) its conformation is rod-like at $L_{c}<l_{p}$ or random coil at $L_{c} l_{p}$. As a rule CMC samples have enough high $M$ and than $L_{c}>l_{p}$; that predicts random coil conformation. This expectation is conformed experimentally by viscometry and light scattering. 
The viscometry measurements of aqueous solutions of cellulose and its derivatives showed out that the factor $a$ in Eq. (16) lies near to $a \approx 0.8$ [29]; this value is characteristic for expanded random coil. The light dependence on the presence of charged or non-charged lateral groups conform the supposition that the CMC rigidity is determined mainly by the $\beta$ 1,4-linkage in the chain backbone; for comparison: $a \approx 0.5$ (defined for non-expanded random coil) for amylose acetate due to $\alpha$-1,4-linkage [26]).

CMC conformation is studied by small angle static light scattering (at eleven fixed angles $\theta=$ $44^{\circ}-151^{\circ}$ ) in combination with size exclusion (gel permutation) chromatography and refractive index detector [8]. That technique allows continuous measuring of $R_{\theta}$ and $c$ while the given fraction is eluted and on-line determining of $\left\langle R_{\mathrm{g}}{ }^{2}\right\rangle$ and $M$ from the initial slope of $c R_{\theta}{ }^{-1}=f\left[M^{-1}, \sin ^{2}(\theta / 2)\right]$ according to Eq. (17). The measurements of CMC samples with degree of substitution DS $=0.75-1.25$ have given $\left\langle R_{\mathrm{g}}^{2}\right\rangle^{1 / 2}=30-140 \mathrm{~nm}$ and mean-weight mass $M_{\mathrm{w}}=120-1100 \mathrm{~kg} / \mathrm{mol}$ (these masses correspond to contour length $L_{\mathrm{c}} \approx 260-2800 \mathrm{~nm}$ ). On Fig. 1 is shown the experimental dependence of $R_{\mathrm{g}}$ on mean-weight $M_{\mathrm{w}}$ or mean-number $M_{\mathrm{n}}$ molecular mass; the difference between $M_{\mathrm{w}}$ and $M_{\mathrm{n}}$ is due to the polydispersity of the polymer samples (the polydispersity index is $M_{\mathrm{w}} / M_{\mathrm{n}} \approx 1.6-2.7$ ).

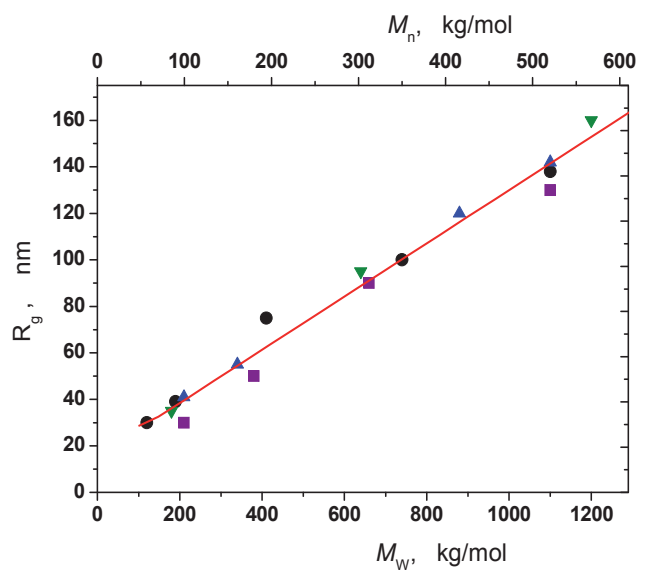

Figure 1. Dependence of the radius of gyration $R_{\mathrm{g}}$ on the mean-weight molecular mass $M_{\mathrm{w}}$ (the bottom abscissa), or mean-number molecular mass $M_{n}$ (the top abscissa) of CMC with DS = $0.75($ ()), 0.91 (7) 0.99 (B) and 1.25 (!). The values of $R_{\mathrm{g}}$ are measured by static light scattering; the data are taken from Ref. [8].

The above cited experimental results allow describing the conformation of CMC chains with $l_{\mathrm{p}} \approx 15-17 \mathrm{~nm}$ at different molecular mass $M$. At $L_{\mathrm{c}} \leq l_{\mathrm{p}}$ the CMC chain has a conformation of bend rod which curvature changes in time due to the thermal motion; the chain with $L_{\mathrm{c}} \approx 15$ - $17 \mathrm{~nm}$ has 29-33 glucose units and $M=3648-4134 \mathrm{~g} / \mathrm{mol}$ (in the case of NaCMC with DS =1.0). At $L_{c}>10 A_{K}$ the CMC chain has a conformation of random coil; a chain 10 times longer than the Kuhn segment $\left(A_{K}=2 l_{\mathrm{p}} \approx 30-34 \mathrm{~nm}\right)$ has 582-660 glucose units and $M=$ $142-160 \mathrm{~kg} / \mathrm{mol}$. So, NaCMC with $M$ higher than $150 \mathrm{~kg} / \mathrm{mol}$ has a conformation of some expanded random coil. 


\subsubsection{Linear charge density and ionic strength}

The viscometry data [29] for high molecular CMC show out that the expansion factor $\alpha_{e}$ in aqueous medium (good solvent for $\mathrm{CMC}, \alpha_{\mathrm{e}}>1$ ) is additionally increased due to the electrostatic repulsion between $\mathrm{COO}^{-}$groups; the effect increases in the range $\mathrm{pH} 2-6(\alpha \approx 1$ at $\mathrm{pH} 7)$ due to the liner charge density $b^{-1}(\alpha)$ increasing with the degree of dissociation $\alpha$. The expansion of the random coil reflects in increased value of the effective persistent length $l_{\mathrm{p}}=l_{\mathrm{p} 0}+l_{\mathrm{pe}}$ and its electrostatic component $l_{\mathrm{pe}}$; the effect is influenced by the ionic strength of the medium. The value of the intrinsic (structural) component $l_{\mathrm{p} 0}$ of the persistent length is estimated to be $5.0 \mathrm{~nm}$ in high salt concentration (where the electrostatic interactions are negligible) [30]. In water/cadoxen ( $\mathrm{Cd}^{2+}$-ethylendiamine complex) mixtures $l_{\mathrm{p} 0}=8.5 \mathrm{~nm}$ was obtained [31].

Static light scattering [8] also show out that the random coil size of CMC depends on the ionic strength; the effect is proportional to the number of Kuhn segments $N_{K}=L_{c}{ }^{2} / 6\left\langle R_{\mathrm{g}}{ }^{2}\right\rangle_{0}$ for chain with contour length $L_{c}$. At $N_{K}=100$ the radius of gyration decreases from $\left\langle R_{\mathrm{g}}\right\rangle^{1 / 2} \approx 190$ $\mathrm{nm}$ (in $0.02 \mathrm{~mol} / \mathrm{L} \mathrm{NaNO}_{3}$ ) to $140 \mathrm{~nm}$ (in $0.1 \mathrm{~mol} / \mathrm{L}$ ). That indicates for diminishing of $\alpha_{e}$ and

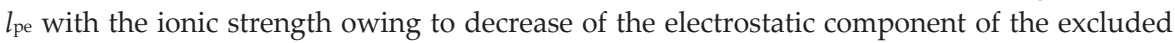
volume (the origin for the random coil expansion).

It seems that the decreasing of $\left\langle R_{\mathrm{g}}{ }^{2}\right\rangle^{1 / 2}$ with salt concentration is caused not entirely by the electrostatic shielding of $\mathrm{COO}^{-}$groups; an additional factor is the structural rigidity $\left(l_{\mathrm{p} 0}\right)$ diminished with dehydration of CMC chain. This effect is probably one of the reasons for high difference (reaching 3 times) of the values of $l_{\mathrm{p} 0}$ obtained at low and high ionic strength; another reason is the imperfections of the theories employing to estimate $l_{\mathrm{p} 0}$ from data obtained by hydrodynamical (viscometry, dynamic light scattering) and statical (static light scattering, potentiometric titration) methods.

At pH $7.0(\alpha \approx 1)$ the light scattering data show out a linear dependence of $\left\langle R_{\mathrm{g}}{ }^{2}\right\rangle^{1 / 2}$ on $M$ in the range $M_{\mathrm{w}}=120-1100 \mathrm{~kg} / \mathrm{mol}$ (Fig. 1); linear is also the dependence of $\left\langle R_{\mathrm{g}}\right\rangle^{1 / 2}$ on the number of statistical segments $N_{K}$ [8]. The both dependences do not depend measurably on the degree of substitution DS $=0.75-1.25$ (in this range the charge parameter $\xi$ increases from 1.04 to 1.73). This independence on DS could be considered as evidence for counterion condensation; i.e. the effective linear charge density $b^{-1}$ does not increase (at $\xi>1$ ) with chain's charges (the number of $\mathrm{COO}^{-}$groups per unit length of $\mathrm{CMC}$ backbone) due to adsorption of $\mathrm{Na}^{+}$ions. This conclusion is done in Ref. [8] but it is not enough reliable because $\left\langle R_{\mathrm{g}}{ }^{2}\right\rangle^{1 / 2}$ of $\mathrm{CMC}$ chain is determined mainly by its structural parameters $\left(l_{\mathrm{p} 0}\right.$ and $\left.L_{c}\right)$ but lightly depends on its electric properties $\left(b^{-1}\right)$ because $l_{\mathrm{p} 0} \gg l_{\mathrm{pe}}$.

\subsection{CMC according to the wormlike chain models}

For $\mathrm{CMC}$ the salt concentration needed to reach the state of unperturbed random coil $(\theta-$ solvent) has been estimated to be about $5 \mathrm{~mol} / \mathrm{L} \mathrm{NaCl}$ [32]. Because this concentration is practically unreachable, the CMC random coil is always expanded. That is why an electrostatic worm-chain theory is needed to evaluate the unperturbed random coil 
parameters, in particular to distinguish the structural and electrostatic components of the excluded volume and the persistent length.

In Ref. [8] the experimental data (obtained by static light scattering) have been used to calculate the CMC molecular parameters according to the models of Odijk [33] and Davis [32]; the last is better validated. The Davis' model gives the following parameters (for CMC with DS $=1.25$ in $0.02 \mathrm{~mol} / \mathrm{L} \mathrm{NaNO}_{3}$ ): (a) expansion factor increasing with $M$ in the range $\alpha_{\mathrm{e}}$ $\approx 1.16-1.40$ at $M_{\mathrm{w}}=120-1100 \mathrm{~kg} / \mathrm{mol}\left(L_{\mathrm{c}}=254-2330 \mathrm{~nm}\right)$; $(b)$ electrostatic component of the persistent length $l_{\mathrm{pe}}=2.9 \mathrm{~nm}$; and $(c)$ intrinsic persistent length (estimated as a fitting parameter) $l_{\mathrm{p} 0}=17 \mathrm{~nm}$. In $0.10 \mathrm{~mol} / \mathrm{L} \mathrm{NaNO}_{3}$ these quantities are: $\alpha_{\mathrm{e}} \approx 1.07-1.14 ; l_{\mathrm{pe}}=0.8 \mathrm{~nm}$; and $l_{\mathrm{p} 0}=15 \mathrm{~nm}[8]$.

In the same work $l_{\mathrm{p} 0} \approx 6 \mathrm{~nm}$ (for $\mathrm{CMC}$ with $\mathrm{DS}=0.9$ in salt-free and in $0.01 \mathrm{~mol} / \mathrm{L} \mathrm{NaCl}$ ) and $l_{\mathrm{p} 0} \approx 3.5 \mathrm{~nm}$ (in $0.1 \mathrm{~mol} / \mathrm{L} \mathrm{NaCl}$ ) was estimated from dependence $\mathrm{pK} \mathrm{a}(\alpha)$ (obtained from titration curves $\alpha(\mathrm{pH})$ ); the values of $l_{\mathrm{p} 0}$ are calculated using the theory of Katchalsky and Lifson [34]. This values are 3-4 fold decreased according to the obtained by Davis's theory (from scattering data for $R_{\mathrm{g}}(M)$ ); the discrepancy reflects rather the imperfections of the theoretical models for wormlike chain than the inaccuracy of the employed experimental methods. It seems that the Davis model gives more realistic values because the KatchalskyLifson model overestimates the electrostatic energy [8].

\subsection{Peculiarity of CMC chain}

The comparison between the intrinsic $l_{\mathrm{p} 0} \approx 15-17 \mathrm{~nm}$ and electrostatic $l_{\mathrm{pe}} \approx 1-3 \mathrm{~nm}$ components of the persistent length (both obtained by Davis model) shows that the high rigidity of $\mathrm{CMC}$ chain is predominantly due to its chemical structure but the electrostatic repulsion plays a minor role although the linear charged density is high at neutral $\mathrm{pH}$ (about 2 charges per $\mathrm{nm}$ ); that leads to relatively low expansion factor $\left(\alpha_{\mathrm{e}} \approx 1.2-1.4\right.$ ) even for very long chains $\left(L_{c}=0.3-2,3 \mu \mathrm{m}\right)$ at low ionic strength.

For comparison: the uncharged chain of poly(ethylene glycol) (PEG) with contour length $L_{c}=$ $26 \mathrm{~nm}$ has $N_{K}=35$ statistical segments with length $A_{K}=0.73 \mathrm{~nm}$, unperturbed radius of gyration $\left\langle R_{\mathrm{g}}{ }^{2}\right\rangle_{0}{ }^{1 / 2} \approx 1.8 \mathrm{~nm}$ and expansion factor $\alpha_{\mathrm{e}}=1.31$ [35]. I.e. due to the flexibility of the PEG chain its Kuhn segment is about 40 times shorter than that of CMC.

\section{Electric polarizability of CMC}

\subsection{Counterions polarizability of free CMC chains}

\subsubsection{Electric polarizability of polyelectrolytes in solution}

When an external electric field with strength $E$ is applied to polyelectrolyte solution an electric polarization $\gamma E$ appears (the coefficient $\gamma$ is named polarizability) [36]. The polarization of polyelectrolytes in aqueous medium is determined mainly by migration of counterions along the chain; this assertion follows from the strong dependence of $\gamma$ on the counterions quantity (determined by the linear charge density $b^{-1}$ and $\mathrm{pH}$-dependent by the degree of dissociation 
$\alpha$ ). The contribution of other kinds of polarization (electronic, atomic and orientation of polar groups) is negligible in fields with moderate strength; a measurable orientation of short rigid chains as DNA fragments is possible only in high voltage electric field [37].

In sinusoidal electric field the frequency $v$ determines the time for which the counterions move in one direction and results in the $\gamma$ magnitude when the half-period is not enough long to reach equilibrium allocation; the critical frequency $v_{1 / 2}$ is defined as $v$ where $\gamma$ decreases twofold. The value of $v_{1 / 2}=1 / 2 \pi \tau_{i}$ is determined by the relaxation time $\tau_{i}$ of the polarization which depends on the distance of migration and the diffusion coefficient of the counterions. So, the polarizability dispersion (the frequency dependence $\gamma=f(v)$ ) gives information for the counterions mobility.

The theories describing the polyelectrolyte polarization (the magnitude of $\gamma$ and its frequency behaviour $\gamma(v)$ ) are based on different physical models considering migration of counterions with diffusion coefficient $D_{i}$ on distance $L$ along a linear polyion. Schwarz's equation [38,39] give $v_{1 / 2}=4 D_{i} / \pi L^{2}$ for strongly elongated particles with length $L$. According Mandel the polarization $\gamma_{i}$ and the relaxation time $\tau_{i}$ of one-dimensional lattice with length $L$ in the case of one type of counterions with valency $z$, mobility $u$ and total number $N$ are [40]:

$$
\gamma_{i}=\left(z^{2} e^{2} / 12 k T\right) N L^{2} \text { and } \tau_{i}=(1 / 12 u k T) L^{2}
$$

where $e, k$ and $T$ are the elementary charge, Boltzmann constant and the absolute temperature.

According Oosawa fluctuation theory the counterions polarization appear in both presence and absence of external electric field; in the last case a spontaneous dipole moments appears due to fluctuation of counterions concentration along the polyelectrolyte chain $[41,42]$. In the case of $N$ number monovalent counterions the main component of the polarizability is:

$$
\gamma_{i}=\left(e^{2} / 2 \pi k T\right) N L^{2} \quad \text { and } \quad \tau_{i}=\left(1 / 4 \pi^{2} u k T\right) L^{2}
$$

The most of the theories consider polyelectrolyte polarization as displacement of one-state counterions or does not distinguish the contribution of the diffuse and condensed counterions. One of the exclusion is Minakata's stochastic theory [43] considering an infinite linear chain of discrete charges which counterions have different transitional mobility depending on the distance from the polyion. The theory shows that the counterions contribution depends on the potential barrier which they have to overcome jumping from one lattice site to the next, so the loosely bound (diffuse) counterions give the main electric polarization at higher frequency; the contribution of strongly bound (condensed) counterions should appear at lower $v$. Thus, the two kinds of counterions are distinguished by their increment to the magnitude of $\gamma$ and by the relaxation frequency $v_{1 / 2}$.

\subsubsection{Polarizability of CMC at random coil conformation}

The counterions polarizability is usually studied by the dielectric permittivity [44] or conductivity $[45,46]$ of polyelectrolyte solutions; the first method is well working in salt-free solution and the second - in small-ions electrolyte. The measuring signal is electrical: the 
capacitance or active current between two electrodes. At these techniques the applied field does not influence the conformation of the polyelectrolyte chain because the field strength is very low and the polarization is due to counterions replacement only. An imperfection of the both methods is that they require high polymer volume fraction because the both polyelectrolyte and the bulk contribute to the measured signal (dielectric or conductivity current); that complicates the interpretation because of the intermolecular interactions at increased polymer concentration. Another problem is the electrode polarization; that limits the ion strength at permittivity measuring.

Dielectric spectroscopy of aqueous solutions of NaCMC shows two dispersions: highfrequency [47] and low-frequency [48]; the presence of two dispersions is typical for polyelectrolytes. The high-frequency dispersion is independent on the contour length but the low-frequency is strongly dependent on it; on this base the two frequency regions are attributed to counterions moving along chain's parts or along the hole polyelectrolyte chain, respectively $[49,50]$.

\subsubsection{Polarizability of CMC at orientation in electric field}

At high field strength the counterions polarization is accompanied with orientation of the polyelectrolyte chain backbone; as a result optical anisotropy of the solution appears. Electro-optical methods are based on registration of the anisotropy of the refractive index $\Delta n$ (birefringence) or the absorption coefficient (dichroism) [51]. A principal advantage of these techniques is that the bulk does not contribute to the measuring signal; that allows multiplying the sensitivity be increasing the optical path and work at lower polymer concentrations. Another important advantage is the possibility to obtain information for both electrical and hydrodynamical properties of the polyelectrolyte chain by measuring the steady-state $\Delta n_{\mathrm{s}}$ and transient $\Delta n_{t}$ anisotropy. These properties can be separately studded by the measuring $\Delta n_{\mathrm{s}} \sim \gamma E^{2}$ and $\Delta n_{t} \sim \Delta n_{\mathrm{s}} \exp \left(-t / \tau_{0}\right)$ with time $t$ after switching off the electric field ( $\tau_{0}$ is the relaxation time determined by the coefficient of friction of macromolecules with given size and form).

The capabilities of the electric birefringence to study aqueous solution of $\mathrm{NaCMC}$ are demonstrated in Ref. [52]. In this work the orientation of CMC with $M=30-250 \mathrm{~kg} / \mathrm{mol}$ is done by applying of a single high voltage rectangular impulse; the field strength reaches $E$ $\approx 2 \mathrm{MV} / \mathrm{m}$. The results show that the polarizability increases with $M$ but the slope of $\gamma=f(L c)$ decreases in the range $M \approx 5-100 \mathrm{~kg} / \mathrm{mol}$ and reaches plateau at $M \approx 250 \mathrm{~kg} / \mathrm{mol}$. The found dependence of $\gamma$ on $L_{c}$ is different from predicted by Eqs. $(17,18)\left(\gamma \sim L^{2}\right)$; the reason for that is revealed comparing the measured disorientation time $\tau_{0}$ with calculated ones for rigid rod, bending rod and worm-like chain. The comparison show out that the experimental values of $\tau_{0}$ corresponds to the rigid rod model $\left(\tau_{0} \sim L^{3}\right)$ only for very short chains with mass less than $M \approx 5 \mathrm{~kg} / \mathrm{mol}$; this $M$ corresponds to 20 glucose residues and $L_{c} \approx 10 \mathrm{~nm}$. This value of $L_{c}$ coincides with the persistent length $l_{\mathrm{p}}=5-15 \mathrm{~nm}$ obtained by viscometry and static light scattering (see section 4.3). 
From the result in Ref. [52] it follows that the counterions are moving along the CMC chain on a distance commensurable with $l_{\mathrm{p}}$. At higher distance the counterions displacement is limited by the local curvature of the chain backbone (which determine the field component along the chain); when the curvature is higher than right angle the counterions are moving in opposite directions along two neighbour chain segments. That means that $l_{\mathrm{p}}$ instead $L_{\mathrm{c}}$ should be used when estimating $\gamma$ and $\tau_{i}$. So, concerning counterions polarizability the long CMC chain can be presented as a consequence of rods likewise the free-articulated chain in the Kuhn model. That allows explaining the experimental fact that $\gamma\left(L_{c}\right)$ is almost constant at $M \approx 250 \mathrm{~kg} / \mathrm{mol}$ [52]; at these $M$ the $\mathrm{NaCMC}$ chain with DS $=1$ has $L_{\mathrm{c}} \approx 530 \mathrm{~nm}$ and about 16 statistical segments with length $A_{\mathrm{K}}=2 l_{\mathrm{p}} \approx 34 \mathrm{~nm}$.

Hydrodynamic behaviour of CMC in electric field suggests that in the case of long chain the orientation of its segments is accompanied by deformation of the random coil; the degree of deformation is determined by both polarizability and rigidity of the chain. The contribution of both electrical and mechanical properties complicates the interpretation of the experimental results about the polarizability of chains with $L_{\mathrm{c}}>l_{\mathrm{p}}$.

Demonstration of that are dependences $\Delta n_{\mathrm{s}}(v)$ of $\mathrm{CMC}$ with 230, 1100 and 2000 glucose units ( $L_{c} \approx 120,570$ and $1030 \mathrm{~nm}$ ) described in Ref. [53]. In this work the sinusoidal field with low strength in combination with very sensitive detection (based on an amplitude modulation of the birefringence signal) is used. The results show out that the dispersion curves $\Delta n_{\mathrm{s}}(v)$ are quite different at frequencies under and up a few kilohertz. In the hertz diapason $\Delta n_{\mathrm{s}}$ has negative values indicating predominant orientation across the electric field; the absolute value $\left|-\Delta n_{\mathrm{s}}\right|$ decreases with diminishing of the degree of dissociation $\alpha$ and with increasing of $M, C_{\mathrm{CMC}}$ and $\mathrm{C}_{\mathrm{NaCl}}$ (concentrations of $\mathrm{CMC}$ and the added $\mathrm{NaCl}$, respectively); $\Delta n_{\mathrm{s}}$ becomes positive (orientation along the field) at high values of $M, C_{\mathrm{CMC}}$ and $C_{\mathrm{NaCl}}$ even at $\alpha=1$. The dependence on $\alpha, M, C_{\mathrm{CMC}}$ and $\mathrm{C}_{\mathrm{NaCl}}$ in kilohertz diapason is analogical although there $\Delta n_{\mathrm{s}}$ is positive in all conditions. The dependence of $\Delta n_{\mathrm{s}}(v)$ on $\alpha$ and $C_{\mathrm{NaCl}}$ in both low and high frequency diapasons confirms that the orientation-deformation of CMC chain is due to counterion polarization. In the same time the dependency of $\Delta n_{\mathrm{s}}(v)$ on $M$ and $C_{\mathrm{CMC}}$ suggest a complicated relation between of the electrically induced conformation of CMC chain and its electrical $(\gamma)$ and mechanical $\left(l_{\mathrm{p}}\right)$ properties; an manifestation of that is the unexpected negative value of $\Delta n_{\mathrm{s}}$. This complexity does not allow application of the theories developed for counterions polarizability $\gamma(L)$ of straight rigid polyions and do very difficult the interpretation of the experimental dependence $\Delta n_{\mathrm{s}}(v)$ in order obtaining information about $\gamma\left(L_{c}\right)$ at $L_{c} \gg l_{p}$.

\subsection{Polarizability of CMC adsorbed on colloid particles}

\subsubsection{Electrophoretic mobility of CMC-alumina particles}

A polyelectrolyte adsorption on solid/liquid interface allows overcoming the difficulties connected with the electrically induced conformation of long CMC chains. In this case the parts of the adsorbed chain are immobilized on the hard surface and that hinders chain deformation. An additional advantage has the adsorption on colloid particles with 
submicron size; then an electric field with moderate strength is enough to orientate the particles due to the their big size (in comparison with the polymer random coil). Then the electro-optical method employed allows registering reliably the orientational degree averaged over the particles ensemble; due to high sensitivity of this technique it is possible to work at low particle concentration.

The adsorption of polyelectrolytes alters the electric and hydrodynamic properties of the solid/liquid interface; an indication for that is the change of the electrophoretic mobility $u$ el of the particles. On Fig. 2 is shown the dependence of $u_{\mathrm{el}}$ on the concentration Cсмс of $\mathrm{NaCMC}$ at adsorption on alumina $\left(\gamma-\mathrm{Al}_{2} \mathrm{O}_{3}\right)$ particles [54]; $u_{\mathrm{el}}=v / E$ is measured by the technique of micro-electrophoresis (microscopic measuring of the velocity $v$ in electric field with strength $E)$. The dependence $u_{\mathrm{el}}\left(\mathrm{C}_{\mathrm{CMC}}\right)$ is studded at $\mathrm{pH} 6$ where the alumina surface is positive (the isoelectric point is at $\mathrm{pH} 8.5$ ) and the $\mathrm{COO}^{-}$groups of $\mathrm{CMC}$ are almost fully dissociated; the opposite charge sign is a precondition for electrostatic adsorption. The change of $u_{\mathrm{el}}$ sign from positive to negative indicates over-equivalent adsorption of CMC and predominant exposition of the polyelectrolyte charges. The steep slope of the curve before the isoelectric point reflects the well known fact that all added polyelectrolyte is adsorbed on the surface at low polymer concentration; that is a characteristic of polyelectrolyte adsorption on an oppositely charged surface $[55,56]$. The plateau at high $\mathrm{C}_{\mathrm{CMC}}$ reflects the saturated adsorption because electrostatic repulsion of already recharged surface and the CMC macromolecules.

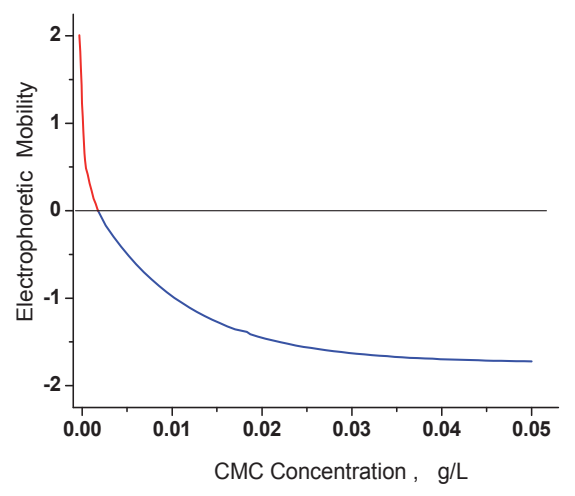

Figure 2. Dependence of electrophoretic mobility $u_{\mathrm{el}}\left[10^{-8}(\mathrm{~m} / \mathrm{s}) /(\mathrm{V} / \mathrm{m})\right]$ of $\gamma-\mathrm{Al}_{2} \mathrm{O}_{3}$ particles on the concentration $\mathrm{C}_{\mathrm{CMC}}[\mathrm{g} / \mathrm{L}]$ of $\mathrm{NaCMC}$ in water-polymer suspension.

The concentration dependence $u_{\mathrm{el}}($ Ссмс) shows out that the surface electric properties of the CMC covered alumina particles are determined predominantly by the charge of the adsorbed layer; the particle surface charge does not manifest itself apparently because of electrostatic and hydrodynamic shielding by the polyelectrolyte layer. This gives opportunity to study electro-optically the electric polarizability of CMC being adsorbed on the colloid particles; in the case the hard core of coated particles determines their optical properties but the orientation depends on the counterions polarization in the polyelectrolyte layer. 


\subsubsection{Electric light scattering in suspension}

The theory of this method [57] is developed in the Rayleigh-Debye-Gans approximation [58] which restricts: (a) the relative refractive index $n=n_{1} / n_{0}\left(n_{1}\right.$ and $n_{0}$ are the refractive indexes of the particles and the medium, respectively); and (b) the relative size $L / \lambda$ (where $L$ is the particle length and $\lambda$ is the wavelength in the medium; $\lambda=\lambda_{0} / n_{0}$ when $\lambda_{0}$ is the wavelength in the vacuum). The light scattering intensity $I_{0}$ at random orientation of the particles is determined by the function of internal interference $P(\theta)$ (form-factor) at scattering angle $\theta$ [27]:

$$
I_{0}=k c H M P(\theta),
$$

where: $k$ - the apparatus constant determined by the scattering volume and the solid angle of the photoreceiver; $c$-the particle weight concentration; $H$ - the optical constant defined by $\lambda_{0}, n_{0}$ and $n_{1} ; M-$ the particle mass.

When an electric field is applied to the suspension, the light scattering intensity $I_{\mathrm{E}}$ is changed due to particles orientation [59]. The absolute electro-optical effect (EOE) $\Delta I=I_{\mathrm{E}}-I_{0}$ can be determined by the functions of internal interference at certain degree of orientation $P(\theta, E)$ and at random orientation $P(\theta)[60]$ :

$$
\Delta I=k c H M[P(\theta, E)-P(\theta)]
$$

At an orientation degree $\Phi$ (varying from 0 at random orientation to 1 at full orientation):

$$
P(\theta, E)=P(\theta)+A(K L) \times \Phi(\gamma, E, T)
$$

where: $K L=2 \pi(L / \lambda) \sin (\theta / 2)$. The optical function $A(K L)$ is determined by the form and relative size $L / \lambda$ of the particles; the expressions for $A(K L)$ are different at low and high degree of orientation. The value of $\Phi$ at steady-state EOE is a function of the electric polarizability $\gamma$, the electric field strength $E$ and the temperature $T$.

The relative EOE $\Delta I / I_{0}$ does not depend on $k, c, H, M$ and it is defined at a moment $t$ only by $P(\theta, E)$ and $P(\theta)$, which are functions of the particles' form, size and optical anisotropy:

$$
\Delta I_{t} / I_{0}=[P(\theta, E) / P(\theta)]-1=[A(K L) / P(\theta)] \times \Phi(\gamma, E, T, t) .
$$

In the steady-state $\Phi(\gamma, E, T)$ depends only on the ratio between the orientation energy $\gamma E^{2}$ and the energy of random motion $k T$. Then EOE at low degrees of orientation $\left(\gamma E^{2}<<k T\right)$ is:

$$
\Delta I_{\mathrm{s}} / I_{0}=[A(K L) / P(\theta)] \times\left(\gamma E^{2} / 15 k T\right) .
$$

The electro-optical results described below are obtained at $\theta=90^{\circ}$ by cell with platinum electrodes at interelectrode distance of $2.6 \mathrm{~mm}$. The light scattering intensities $I_{0}$ (in absence of electric field) and $\Delta I_{\mathrm{s}}$ (in sinusoidal field with strength $E \leq 54 \mathrm{kV} / \mathrm{m}$ ) were measured after reaching steady-state polymer adsorption. 


\subsubsection{Kilohertz polarizability of CMC-alumina particles}

Figures 3 and 4 represent the field dependence $\Delta I / I_{0}=f\left(E^{2}\right)$ at several CMC concentrations. The linearity of the graphics means that the degree of orientation is low, i.e. $\gamma E^{2}<<k T$. Therefore $\gamma$ is proportional to the slope of the lines $\left[\left(\Delta I / I_{0}\right) / E^{2}\right]$ according to eq. (23).

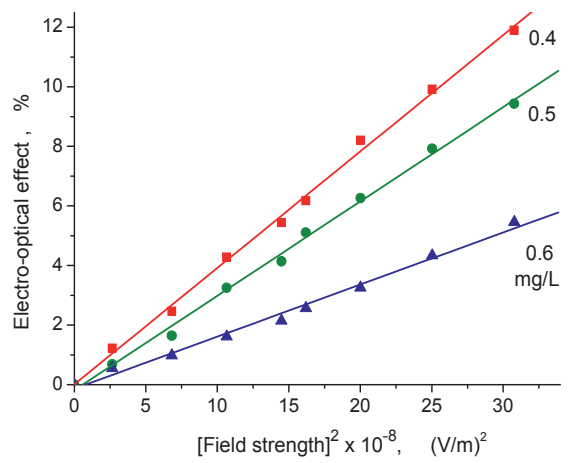

Figure 3. Dependence of steady-state EOE $\left(\Delta I_{s} / I_{0}\right) \times 100$ in water-polymer suspension of $\gamma-\mathrm{Al}_{2} \mathrm{O}_{3}$ particles on the squared field strength $E^{2}\left[10^{8} \mathrm{~V}^{2} \mathrm{~m}^{-2}\right]$ at $1 \mathrm{kHz}$ and $\mathrm{NaCMC}$ concentrations $C_{\mathrm{CMC}}=(4-6) \times 10^{-4} \mathrm{~g} / \mathrm{L}$ $\mathrm{NaCMC}$ (under the recharging point).

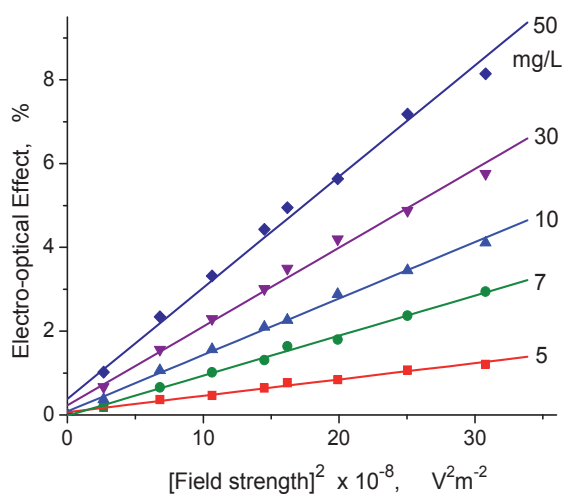

Figure 4. Field strength dependence (the same as in Fig. 3) at $\mathrm{C}_{\mathrm{CMC}}=(5-50) \times 10^{-3} \mathrm{~g} / \mathrm{L} \mathrm{NaCMC}$ (above the recharging point).

Fig. 3 shows out that the polarizability decreases with CMC concentration but Fig. 4 demonstrates just the opposite: $\gamma$ increases with Ссмс. The both trends correlate with the concentration dependence of the electrophoretic mobility $u_{\mathrm{el}}($ (Смс) (Fig. 2) which reflects the alteration in the total surface charge of the particles with adsorbed CMC. That means that 
the magnitude of $\gamma$ depends mainly on the total charge of the diffuse counterions (which decreases before the recharging point and increases after it). So, the kilohertz polarizability is due predominatingly to the diffuse counterion but that does not exclude participation of condensed ions.

\subsubsection{High frequency dispersion of CMC-alumina polarization}

The change of the half-period $1 / 2 v$ of the applied sinusoidal electric field allows altering the ions migration distance and by that the contribution to $\gamma$ of the counterions being in two physical states: adsorbed and diffuse. According the theory (see paragraph 5.1.1) the condensed cations have diminished mobility along the $\mathrm{CMC}$ chain due to the strong electrostatic interaction with the charged $\mathrm{COO}^{-}$groups. The critical frequency $v_{1 / 2} \sim D_{i} / L^{2}$ depends on the polyion length $L$ and the diffusion coefficient $D_{i}$ of the counterions electrically associated to the polyelectrolyte chain (Eqs. 17, 18); so, at constant $L$ (absence of particles aggregation, see Ref. [54]) the value of $v_{1 / 2}$ gives information about the counterions mobility. The dispersion dependence $\gamma(v)$ shows the magnitude and $v_{1 / 2}$ of the polarizability components with different $D_{i} / L$; that allow distinguishing the contribution of the condensed and diffuse counterions.

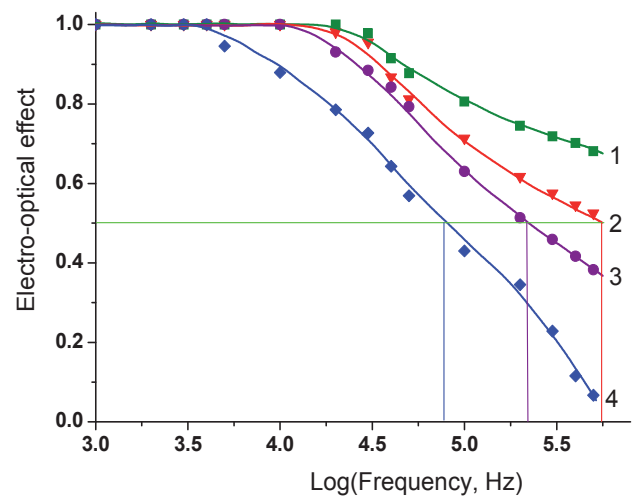

Figure 5. Normalized high frequency dependence of EOE $\left(\Delta I / I_{0}\right)$ of alumina particles in water (curve 1) and in aqueous solution NaCMC $\left(M_{\mathrm{w}}=250 \mathrm{~kg} / \mathrm{mol}\right)$ at concentrations: $4 \cdot 10^{-4} \mathrm{~g} / \mathrm{L}$ (curve 2$), 6 \cdot 10^{-4} \mathrm{~g} / \mathrm{L}$ (curve 3) and $1 \cdot 10^{-2} \mathrm{~g} / \mathrm{L}$ (curve 4 ). The EOE $\Delta I_{\mathrm{s}} / I_{0}$ at $1 \mathrm{kHz}$ is taken to be one for all curves.

The frequency dependences of EOE of alumina particles with size $0.3 \mu \mathrm{m}$ in CMC solution is shown in Fig. 5. The total particle charge is positive for curves 1-3 and negative for curve 4 (recharged surface). The critical frequency decreases with the adsorbed CMC amount: $v_{1 / 2}$ $\approx 550 \mathrm{kHz}$ (curve 2), $v_{1 / 2} \approx 220 \mathrm{kHz}$ (curve 3) and $v_{1 / 2} \approx 80 \mathrm{kHz}$ (curve 4).

The form of the dispersion curves $\gamma(v)$ indicates that the polarization is due to one kind of counterions; in the case of different $D_{i} / L$ the curves should have a shoulder with fixed $v_{1 / 2}$ but increasing increment while the CMC adsorbed amount grows. The value of $v_{1 / 2}$ at 
saturated adsorption (curve 4) is not very different from that at small CMC surface concentration (curves 3 and 2) and bare particle (curve 1) determined by the diffuse counterions polarization; this fact together with the absence of noticeable shoulder allows concluding that the polarizability of the CMC covered surface (curve 4) is due to the diffuse counterions around the particles. The displacement of the $v_{1 / 2}$ to lower frequencies could be explained with their decreased mobility because of the presence of polymer layer, i.e. the effective diffusion coefficient $D_{i}$ is smaller than that of free ions in solution.

Nevertheless, the results in Fig. 5 do not exclude unambiguously the condensed counterions participation in the polarization. The absence of the increasing shoulder at CMC adsorption could be owing to equal ratio $D_{i} / L$ for the condensed and diffuse counterions. In this case $D_{\mathrm{Na}}$ of the condensed $\mathrm{Na}^{+}$ions should be 400 times less than that of the diffuse ones assuming $L=l_{\mathrm{p}}=15 \mathrm{~nm}$ for bound counterions and $L=0.3 \mu \mathrm{m}$ (the particle size) for the free ones.

To exclude the possibility that the values of $v_{1 / 2} \sim D_{i} / L^{2}$ of the diffuse and condensed counterions polarization coincide due to unique combination of $L$ and $D_{i}$ we have studied the concentration dependence $\gamma($ Ссмс) which reflects the magnitude of $\gamma$ as function of the quantity of both kinds of counterions [54]. The measurements are performed at $v=1 \mathrm{kHz}$ where the half-period $1 / 2 v$ is long enough to reach the equilibrium distribution of counterions in any moment $t$ in a field with instantaneous strength $E_{\mathrm{t}}=E_{0} \sin (2 \pi v t)$; indication for that is the plateau at all С СмС (Fig. 5).

\subsubsection{Concentration dependence of CMC-alumina polarizability}

The contribution of the diffuse and condensed counterions can be estimated by comparing the concentration dependences of the polarizability $\gamma(\text { Ссмс })_{\text {and mobility }} u_{\mathrm{el}}\left(\mathrm{C}_{\mathrm{CMC}}\right)$. This approach is based on the fact that the condensed counterions move together with the polyelectrolyte chain in a permanent electric field, that is why they are not seen in methods based on the translational motion in a d.c. electric field [61]. I.e. the $u$ el gives information about the quantity of the diffuse counterions only while the polarizability in a sinusoidal field reflects participation of the all movable counterions.

When the amount of adsorbed CMC increases with Ссмс then the ratio condensed/diffuse counterions alters but in a different way before and after the isoelectric point. The quantity of condensed ions increases for all С Смc concentrations where adsorption appears but the quantity of the diffuse counterions decreases before the recharging point and increases after it. The comparison between $\gamma($ С $\mathrm{CMC})$ and $u_{\mathrm{el}}\left(\right.$ С $\left._{\mathrm{CMC}}\right)$ at lower С $_{\mathrm{CMC}}$ concentrations can give unambiguous information about the relative contribution of the diffuse and condensed counterions due to the opposite change of their quantity. In this concentration range the slope of the dependence $\gamma\left(\right.$ С $\left._{\mathrm{CMc}}\right)$ should be negative but smaller than that of $u_{\mathrm{el}}\left(\mathrm{C}_{\mathrm{CMC}}\right)$ taking into account that the adsorbed CMC introduces approximately one condensed and two diffuse $\mathrm{Na}^{+}$counterions on every three $\mathrm{COO}^{-}$groups (see paragraph 3.3.5). 
Fig. 6 refutes this expectation: both the curves have the same slope before the isoelectric point; this disproves the hypothesis that the condensed ions participate in the polarization. The difference at the higher $\mathrm{CMC}$ concentrations can be explained by the different influence of the polymer layer on the behaviour of the counterions in direct and sinusoidal electric field and by the way the figure is constructed: the two quantities are equated $\left(\gamma=u_{\text {el }}\right)$ at $\mathrm{C}_{\mathrm{CMC}}=0$.

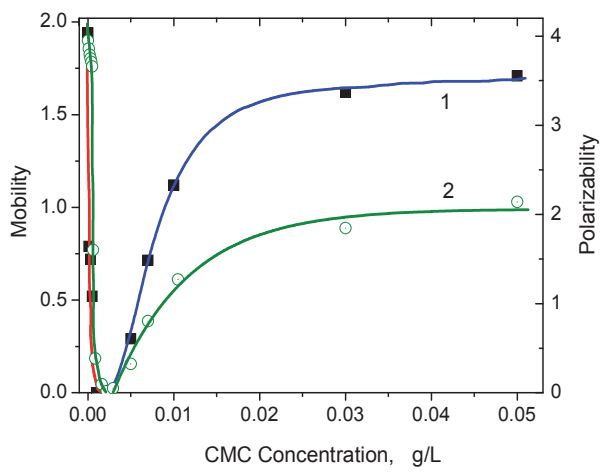

Figure 6. Fig. 6. Dependences of the absolute value $\left|u_{\mathrm{el}}\right|$ of the electrophoretic mobility (curve 1, left ordinate) and the electric polarizability $\gamma$ (curve 2, right ordinate) of $\gamma-\mathrm{Al}_{2} \mathrm{O}_{3}$ particles on the concentration $\mathrm{C}_{\mathrm{CMC}}$ of $\mathrm{NaCMC}$. The $u_{\mathrm{el}}$ and $\gamma$ are given in relative units assumed to be equal in the absence of CMC. The sign of $u_{\mathrm{el}}$ is positive at CСмC under $3 \mathrm{mg} / \mathrm{L}$ (the red part of the curve) and negative above this concentration (the blue part).

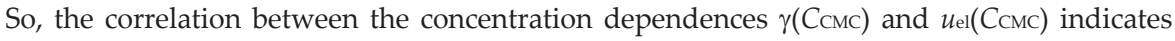
that the kilohertz polarizability of the alumina particles with adsorbed CMC is determined by the diffuse counterions.

\subsubsection{Comparing with the literature}

A low frequency shift of the polarizability with $v_{1 / 2} \approx 3 \mathrm{kHz}$ is found at saturated CMC adsorption on ferrioxide particles [62]. That value of $v_{1 / 2}$ is 25 times lower than ours (curve 4 on Fig. 5) although in both experiments the same CMC specimen $\left(M_{\mathrm{w}}=250 \mathrm{~kg} / \mathrm{mol}\right.$, DS $=1.2$ ) is studied. The difference in $v_{1 / 2}$ could be related to the different particles employed; this suggests that the value of $v_{1 / 2}$ is determined by $L$ rather than by $D_{i}$. An additional argument is that the $v_{1 / 2} \approx 80 \mathrm{kHz}$ of CMC coated alumina particles lies in the frequency region specific for the diffuse counterions polarizability of elongated ellipsoids with $L \approx 0.3$ $\mu \mathrm{m}$ (taking into account that $v_{1 / 2} \sim 1 / L^{2}$ ) [63]. That means that the interfacial polarization of CMC coated particles is determined by the diffuse counterions displacement around the whole particle but not along the CMC chains. If the bound counterions are the origin of the electric polarization then a shoulder on the dispersion curve should appear and its increment must be proportional to the surface CMC amount. 
In Ref. [62] a principally different conclusion is drawn: the electric polarizability of CMC coated particles is due to the condensed counterions. The interpretation is based on the lowfrequency shift of the dispersion curves employing the Schwarz's equation $v_{1 / 2}=4 D_{i} / \pi L^{2}$ and using the literature value $D_{i}=6 \cdot 10^{-11} \mathrm{~m}^{2} / \mathrm{s}$ [64] for bound $\mathrm{Na}^{+}$in a CMC solution; the CMC chain is assumed to be a straight polyion with length $L=505 \mathrm{~nm}$ (equal to the contour length $L_{c}$ of $C M C$ with $M=250 \mathrm{~kg} / \mathrm{mol}$ ). Then, accepting that the estimated $v_{1 / 2}=300 \mathrm{~Hz}$ is close to the experimentally obtained $v_{1 / 2} \approx 3 \mathrm{kHz}$, the authors conclude that the orientation of CMC coated particles in the electric field is due to condensed counterions polarization along the adsorbed polyelectrolyte chains.

The interpretation in Ref. [62] is wrong because the CMC chain with a contour length $L_{c}=0.5$ $\mu \mathrm{m}$ cannot be considered as rod-like neither free in the solution nor adsorbed on the particle surface. It is well known that parts of adsorbed chain take a quasi-planar (two-dimensional) conformation and the rest of the chain retains a three-dimensional conformation [65], so the form of adsorbed CMC is predetermined by its conformation in solution. A free CMC chain with $M=250 \mathrm{~kg} / \mathrm{mol}$ has the conformation of a random coil with $N_{K} \approx 15$ statistical segments (see section 4.3) and a radius of gyration $R_{\mathrm{g}}=44 \mathrm{~nm}$ (Fig. 1). So, for a CMC chain with $L_{\mathrm{c}}=0.5$ $\mu \mathrm{m}$ the magnitude of $\gamma$ and the critical frequency $v_{1 / 2}$ cannot be estimated using the contour length $L_{c}$ in the equations derived for the polarizability of straight polyions. This conclusion is confirmed by results for CMC in aqueous solution: the experimental dependence $\gamma\left(L_{c}\right)$ does not correspond to $\gamma \sim L_{c}^{2}$ predicted by Eq. $(17,18)$ but has a plateau at $M \geq 200 \mathrm{~kg} / \mathrm{mol}$ [52]; so, the dependence $v_{1 / 2} \sim L^{-2}$ (according Schwarz's equation) is also not applicable for long CMC chains.

My opinion is that the polarizability of the polymer covered particles is due to the diffuse counterions but their mobility is decreased because of the presence of the polymer layer, i.e. the effective diffusion coefficient $D_{i}$ is smaller than that of the free ions in solution. The reason for such interpretation is the fact that the dispersion curve displacement (towards lower $v_{1 / 2}$ ) increases with the CMC amount on the particle surface.

\section{Author details}

Alexandar Metodiev Zhivkov

Institute of Physical Chemistry, Bulgarian Academy of Sciences, Acad. G. Bonchev Str., Sofia, Bulgaria

\section{References}

[1] D.A. Rees, Adv. Carbohydr. Chem. Biochem. 24 (1969) 267-332.

[2] V.S.R. Rao, N. Yathindra, P.R. Sundararajan, Biopolymers 8 (1969) 325-333.

[3] B.K. Sathyanarayana, V.S.R. Rao, Biopolymers 11 (1972) 1379-1394.

[4] E. Ott, H.M. Spurlin, M.W. Grafflin, Cellulose and Cellulose Derivatives, part II, High Polymers, vol. V, Interscience publishers, New York, 1954. 
[5] D.E. Metzler, Biochemistry, Academic press, New York, 1977.

[6] J. Reuben, H.T. Connor, Carbohydr. Res. 115 (1983) 1-13.

[7] Z. Ma, Z. Wiebang, Z. Li, Chinese J. Polym. Sci. 7 (1989) 45.

[8] C.W. Hoogendam, A. de Keizer, M.A. Cohen Stuart, B.H. Bijsterbosch, J.A.M. Smit, J.A.P.P. van Dijk, P.M. van der Horst, J.G. Batellaan, Macromolecules 31 (1998) 6297-6309.

[9] A. Katchalsky, N. Shavit, H. Eisenberg, J. Polym. Sci. 13 (1954) 69-84.

[10] F.H. Crowdhury, S.M. Neale, J. Polym. Sci. 1 (1963) 2881.

[11] L. Zhang, T. Takematsu, T. Norisuye, Macromolecules 20 (1987) 2882.

[12] A.V. Dobrynin, M. Rubinstain, Prog. Polym. Sci. 30 (2005) 1049-1118.

[13] A. Katchalsky, Z. Alexandrowicz, In: B.E. Conway, R.G. Baradas (Eds.), Chemical physics of ionic solutions, London, Wiley, 1966. p.295-346.

[14] F. Oosawa, Polyelectrolytes, New York, Marcel Dekker, 1971.

[15] R.M. Fuoss, A. Katchalsky, S. Lifson, Proc. Natl. Acad. Sci. USA 37 (1951) 579-586.

[16] Z. Alexandrowicz, A. Katchalsky, J. Polym. Sci. Part A 1 (1963) 3231-3260.

[17] A. Katchalsky, Pure Appl. Chem. 26 (1971) 327-374.

[18] T. Nishio, A. Minakata, J. Chem. Phys. 113 (2000) 10784-10792.

[19] T. Nishio, A. Minakata, J. Phys. Chem. B, 107 (2003) 8140-8145.

[20] H. Ohshima, K. Furusawa (eds.), Electrical Phenomena at Interfaces: Fundamentals, Measurements and Applications, 2ed edition, Dekker, New York, 1998.

[21] H. Ohshima, Theory of Colloid and Interfacial Electric Phenomena, Elsevier/Academic Press, Amsterdam, 2006.

[22] G.S. Manning, J. Chem. Phys. 51 (1969) 924-933.

[23] G.S. Manning, Polyelectrolyte binding, 12 (1979) 443-449.

[24] H. Ohshima, Biophysical Chemistry of Biointerfaces, Wiley, 2010.

[25] C.R. Cantor, P.R. Schimmel, Biophysical Chemistry, W.H. Freeman and Company, San Francisco, 1980.

[26] S.R. Rafikov, V.P. Budtov, Y.B. Monakov, Introduction in physico-chemistry of polymer solutions, Nauka, Moscow, 1978.

[27] B.E. Eskin, Light Scattering by Polymer Solutions and Macromolecule Properties, Nauka, Leningrad, 1986.

[28] B.E. Eskin, Scattering of Light by Polymer Solutions, Nauka, Moscow, 1973.

[29] M. Yalpani, Polysaccharides, Elsevier, New York, 1988.

[30] M. Rinaudo, in: J.F. Kennedy, G.O. Phillips, P.O. Williams, L. Piculell (eds), Cellulose and cellulose derivates: physicochemical aspects and industrial applications, Woodhead Publishing Limited, Cambrige, U.K., 1995.

[31] P.N. Lavrenko, O.V. Okatova, V.N. Tsvetkov, H. Dautzenberg, B. Philipp, Polymer 31 (1990) 348-352.

[32] R.M. Davis, Macromolecules 24 (1991) 1149-1155.

[33] T. Odijk, Biopolymers 18 (1979) 3111-3113.

[34] A. Katchalsky, S. Lifson, J. Polym. Sci. 13 (1954) 43-55.

[35] A.M. Zhivkov, J. Colloid Interface Sci. 113 (2007) 122-127. 
[36] S.S. Dukhin, V.N. Shilov Dielectric Phenomena and Double Layer in Disperse Systems and Polyelectrolytes, Wiley, New York, 1974.

[37] D. Porschke, M. Antosiewicz, in: S.P. Stoylov and M.V. Stoimenova (eds.), Molecular and Colloid Electro-optics, Taylor \& Francis, New York, 2007.

[38] G. Schwarz, J. Phys. Chem. 66 (1962) 2636-2641.

[39] M. Mandel, A. Jenard, Transactions Faraday Soc. 59 (1963) 2158-3169.

[40] M. Mandel, Mol. Phys. 4 (1961) 489-496.

[41] F. Oosawa, Biopolymers 9 (1970) 677-688.

[42] A. Minakata, N. Imai, F. Oosawa, Biopolymers 11 (1972) 347-354.

[43] A. Warashina, A. Minakata, J. Chem. Phys. 58 (1973) 4743-4749.

[44] M. Mandel, in: S. Krause (Ed.) Molecular Electro-Optics, Plenum Press, New York, 1981.

[45] A. Minakata, H. Takahashi, T. Nishio, J. Nagaya, A. Tanioka, Colloids \& Surfaces A:209 (2002) 213-218.

[46] A. Minakata, T. Nishio, M. Kimura, S.-I. Yano, Y. Tanaka, T. Shimizu, Colloids \& Surfaces B: Biointerfaces 56 (2007) 277-280.

[47] L.-G. Allgen, S. Roswall, J. Polymer Sci. 12 (1954) 229-236.

[48] A. Oga, A. Takimoto, R. Hayakawa, Y. Wada, Rept. Progr. Polymer Phys. Japan, 24 (1981) 77.

[49] M. Mandel, Ann. New York Acad. Sci. 303 (1977) 74-87.

[50] Th. Vreugdenhil, F. van der Touw, M. Mandel, Biophys. Chem. 10 (1979) 67-80.

[51] Fredericq E., Houssier C., Electric Dichroism and Electric Birefringence, Clarendon press, Oxford, 1973.

[52] A.R. Foweraker, B.R. Jennings, Polymer 16 (1975) 720-724.

[53] T. Yamamoto, Y. Mori, N. Ookubo, R. Hayakawa, Y. Wada, Colloid \& Polymer Sci. 260 (1982) 20-26.

[54] A.M. Zhivkov, R.P. Hristov, J. Physics: Condensed Matter 22 (2010) 494112 (7 pp).

[55] J. Gregory, S. Barany, Advances Colloid. Interface Sci., 169 (2011) 1-12.

[56] F. Bordi, S. Sennato, D. Truzzolillo, J. Phys: Condens. Matter 21 (2009) 203102 (26 pp.).

[57] S.P. Stoylov, Colloid Electro-optics - Theory, Techniques and Applications, Acad. Press, London, 1991.

[58] H.C. van de Hulst, Light Scattering by Small Particles, John Wiley, New York, 1957.

[59] A.A. Spartakov, A.A. Trusov, A.V. Voitylov, V.V. Vojtylov, in: S.P. Stoylov and M.V. Stoimenova (eds.), Molecular and Colloid Electro-optics, Taylor \& Francis, New York, 2007.

[60] A.M. Zhivkov, in: S.P. Stoylov and M.V. Stoimenova (eds.), Molecular and Colloid Electrooptics, Taylor \& Francis, New York, 2007.

[61] M.C. Stuart, R. de Vries, H. Lyklema, Polyelectrolytes, in: Lyclema H. (Ed.), Fundamentals of interface and colloid science, vol. V, Elsevier, London, 2005.

[62] Ts. Radeva, K. Kamburova, J. Colloid Interface Sci. 293 (2006) 290-295.

[63] V.N. Shilov, Yu.B. Borkovskaja, S.N. Budankova, in: S.P. Stoylov, M. Stoimenova (eds.), Molecular and Colloid Electro-optics, Taylor \& Francis, New York, 2007.

[64] N. Oocubo, Y. Hirai, K. Ito, R. Hayakawa, Macromolecules 22 (1989) 1359-1366. 
[65] G.J. Fleer, M.A. Cohen-Stuart, J.M.H.M. Scheutjens, T. Cosgove, B. Vincent, Polymers at interfaces, London, Chapman \& Hall, 1993. 